\title{
A Holistic Solution for Reliability of 3D Parallel Systems
}

\author{
JAVAD BAGHERZADEH, APORVA AMARNATH, JIELUN TAN, SUBHANKAR PAL, \\ and RONALD G. DRESLINSKI, University of Michigan, Ann Arbor, MI
}

\begin{abstract}
Monolithic 3D technology is emerging as a promising solution that can bring massive opportunities, but the gains can be hindered due to the reliability issues exaggerated by high temperature. Conventional reliability solutions focus on one specific feature and assume that the other required features would be provided by different solutions. Hence, this assumption has resulted in solutions that are proposed in isolation of each other and fail to consider the overall compatibility and the implied overheads of multiple isolated solutions for one system.

This article proposes a holistic reliability management engine, R2D3, for post-Moore's M3D parallel systems that have low yield and high failure rate. The proposed engine, comprising a controller, reconfigurable crossbars, and detection circuitry, provides concurrent single-replay detection and diagnosis, fault-mitigating repair, and aging-aware lifetime management at runtime. This holistic view enables us to create a solution that is highly effective while achieving a low overhead. Our solution achieves $96 \%$ coverage of defect; reduces $V_{t h}$ degradation by $53 \%$, leading to a $78 \%$ performance improvement on average over 8 years for an eight-core system; and ultimately yields a $2.16 \times$ longer mean-time-to-failure (MTTF) while incurring an overhead of $7.4 \%$ in area, $6.5 \%$ in power, and an $8.2 \%$ decrease in frequency.
\end{abstract}

CCS Concepts: • Hardware $\rightarrow$ Emerging architectures; 3D integrated circuits; Aging of circuits and systems;

Additional Key Words and Phrases: Monolithic 3D circuits, hard fault, fault detection, aging, 3-d circuits

\section{ACM Reference format:}

Javad Bagherzadeh, Aporva Amarnath, Jielun Tan, Subhankar Pal, and Ronald G. Dreslinski. 2021. A Holistic Solution for Reliability of 3D Parallel Systems. J. Emerg. Technol. Comput. Syst. 18, 1, Article 23 (November 2021), 27 pages.

https://doi.org/10.1145/3488900

\section{INTRODUCTION}

With the decline of Moore's law, Monolithic 3D (M3D) integration opens up the possibility of designing cores and associated networks using multiple tiers by utilizing monolithic inter-tier vias (MIVs) [40] and, hence, reducing the effective wire length [25]. Compared to Through Silicon Via (TSV)-based 3D ICs [30], M3D offers the "true" benefits of vertical dimension for system integration thanks to the extremely small size of inter-tier vias. Architects have leveraged the performance benefits of this vertical stacking to significantly decrease the runtime of computeintensive workloads by stacking multiple layers of cores/processors to create 3D parallel systems

Authors' address: J. Bagherzadeh, A. Amarnath, J. Tan, S. Pal, and R. G. Dreslinski, University of Michigan, 2260 Hayward Street, Ann Arbor, MI, 48109; emails: \{javadb, aporvaa, jieltan, subh, rdreslin\}@umich.edu.

Permission to make digital or hard copies of all or part of this work for personal or classroom use is granted without fee provided that copies are not made or distributed for profit or commercial advantage and that copies bear this notice and the full citation on the first page. Copyrights for components of this work owned by others than ACM must be honored. Abstracting with credit is permitted. To copy otherwise, or republish, to post on servers or to redistribute to lists, requires prior specific permission and/or a fee. Request permissions from permissions@acm.org.

(c) 2021 Association for Computing Machinery.

1550-4832/2021/11-ART23 \$15.00

https://doi.org/10.1145/3488900 


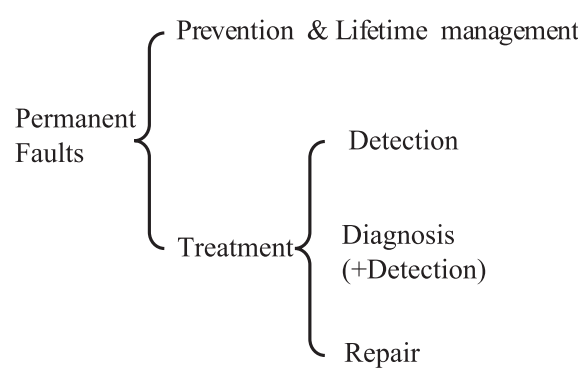

NBTI 3D[34], Bubblewrap[27], NBTI

multilcore[52], Facelift[54],

Proactive[38], Arthemis[4]

ARGUS[36], ACE[7],

mSWAT[19], DIVA [2],

Online testing[47]

Core Cannibal[45], 3DFAR[3], StageNet[17], Vipor[42], Cobra[41]

\section{R2D3}

Fig. 1. Required features for holistic reliability solution and existing solutions are built in isolation for a particular category.

for data accelerators [15, 32] and Network-on-Chip (NoC) architectures [2, 13, 21, 58, 62]. Fabrication of the first monolithic 3D IC within a foundry has been a great leap toward the realization of such architectures [1]. However, despite the benefits offered, yield and reliability are still the major obstacles for commercial realization of this technology [16, 24, 27, 54]. Processors in 3D technology are more susceptible to permanent transistor failures, often due to wearout phenomena such as time-dependent dielectric breakdown (TDDB) [33], negative bias temperature instability (NBTI) [10], and electromigration (EM) [12]. Recent studies have shown how the elevated temperatures and longer heat dissipation paths in 3D ICs lead to significantly rapid aging and higher fault rates [27, 29, 31, 43, 54]. Hence, to commercialize this new technology, new architectural and circuit modifications that can work around high fault rates are required, improving performance comparable to Silicon, while the manufacturing process is perfected [3].

As shown in Figure 1, previous solutions can be divided into two categories: prevention-methods that slow down aging by decelerating wearout, and treatment-methods that deal with the failures once they have occurred. Prevention methods affect wearout or failure mechanisms, such as $V_{t h}$ degradation, by controlling parameters like temperature, power, utilization, workload, frequency, and supply voltage. Treatment methods are designed to tolerate faults encountered during operation. As shown in Figure 1, a reliable treatment system requires the inclusion of three critical capabilities: (1) detection to identify the presence of a fault; (2) diagnosis to locate the source of the fault, i.e., to find the faulty component(s); and (3) repair to isolate the failure from the system [17]. These three characteristics of treatment, combined with prevention, are the four pillars of a holistic reliability solution.

Challenges associated with low yield and high fault rates of the 3D technology call for the incorporation of both prevention and treatment mechanisms in one solution. Previous solutions, as have been included in Figure 1, focus on one specific pillar of reliability and provide remedy for that issue. These solutions are usually proposed in isolation of each other, failing to take the implications of other aspects into account. Although the mentioned design approach can help to break down the problem, a narrow design perspective leads to solutions that are difficult to deploy in practice. The other issue with the previous approaches is that most of these solutions are proposed for a particular architecture and are not compatible with each other, so it is impossible or extremely difficult to combine them to create a holistic solution. Even if we ignore the compatibility issues and forcefully combine multiple solutions in a Frankenstein's Monster-like solution, the overall overhead will be too high. A more detailed comparison to these prior works is in Section 6 .

This article proposes Reliability by Reconfiguring 3D systems-R2D3, a holistic, agingaware reliability engine with fine-grained reconfigurability for parallel streaming systems that can concurrently detect, diagnose, repair, and prevent failures at runtime. The engine, comprising 
a controller, reconfigurable crossbars, and detection circuitry, takes advantage of the smaller routing delay over vertical layers in M3D circuits to create a fast-reconfigurable fabric that leverages the availability of identical resources in parallel systems. Figure 5 shows high-level components of R2D3 incorporated into an in-order core-based parallel 3D system where vertical buses that act as crossbars are inserted between consecutive stages. Our controller creates epochs of execution, at the end of which the detection circuitry can access the inputs and outputs of all layers to verify correctness and diagnose faults. Instead of adding extra redundancy, we salvage leftovers, which are the functional pipeline stages from faulty cores for fault detection and prevention (orange stages in Figure 2). The R2D3 controller manages the reconfigurable fabric to concurrently provide single-cycle replay detection and diagnosis, fault-mitigating repair, and aging-aware lifetime management during runtime. To prevent faults, the controller considers temperature variation across the chip and activity factor of each stage to adaptively reconfigure functioning stages to create virtual pipelines. This balances out the aging rate, extending the lifetime of the system. R2D3 can be adopted to M3D parallel architectures that have a large number of identical units, such as systolic arrays, mesh-based systems, and many-core or multi-core systems. In this work, we adopt R2D3 for vertically stacked in-order (OpenSPARC T1) and out-of-order (OoO) pipelines (ARM Cortex A9), as such pipelines closely resemble the basic compute units in modern-day streaming accelerators and massively parallel systems.

We evaluate R2D3 on a physical design of an eight-core, in-order OpenSPARC T1 processor, modified to support fine-grained reconfiguration in $3 \mathrm{D}$, to treat and prevent faults caused by aging, which is analyzed by studying the thermal implications. We compare the following eight-core 3D systems: (1) system with R2D3 engine using an adaptive and dynamic reconfiguration policy (R2D3-Pro), (2) R2D3 engine using a round-robin dynamic non-adaptive reconfiguration policy (R2D3-Lite), (3) system equipped with failure-repairing static reconfiguration policy (Static), and (4) a 3D-stacked processor with no reconfiguration infrastructure (NoRecon). Our evaluation shows that our fault detection technique provides a high coverage of silicon defects (96\%). R2D3Lite and R2D3, respectively, achieve a $1.63 \times$ and $2.16 \times$ improvement in lifetime and $52 \%$ and $78 \%$ increase in throughput over NoRecon, while incurring a marginal $7.4 \%$ area and $8.2 \%$ frequency overhead in comparison to the NoRecon design and negligible overhead in comparison to Static. Furthermore, R2D3 reduces $V_{t h}$ degradation by $53 \%$ over a period of 8 years in comparison to NoRecon and Static.

In summary, this article makes the following contributions:

(1) R2D3 demonstrates how a robust and practical solution should synergistically support all four pillars of reliability: prevention, detection, diagnosis, and repair of failures at a low overhead with smart reuse and management of the underlying reconfigurable 3D fabric.

(2) We propose R2D3 as a reliability engine that can detect and diagnose faults by re-executing instructions on idle units, incurring a small performance and area overhead. It detects transient and permanent faults and distinguishes them by using a single-cycle replay, while localizing the fault to the granularity of a pipeline stage.

(3) R2D3 prevents failures by introducing graceful degradation using smart scheduling policies on the same reconfigurable architecture without any additional overhead in $3 \mathrm{D}$. It considers the activity factor of each stage along with its temperature variation by adaptively creating virtual pipelines that balance out the aging rate.

\section{MOTIVATION}

The failure rate for electronic systems follows the bathtub curve [51], which describes the probability of permanent failure spanning three distinct periods: early failures (fabrication, packaging, 


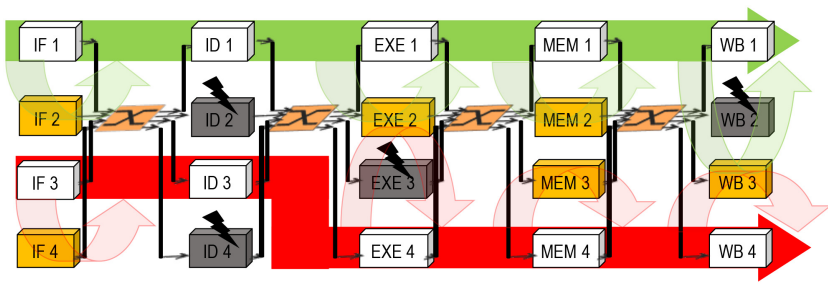

Fig. 2. Schematic of the R2D3 Engine, where corresponding pipeline stages are stacked vertically and crossbars are inserted between consecutive stages. In this four-fault situation, our solution dynamically reconfigures to connect healthy units as shown with the red and green stripes, providing the compute power of two complete cores. Stages in orange are leftovers and still functional and are swap-out working stages to distribute wearout and to detect, diagnose, and repair faults.

and shipping), random failures, and wearout failures. Although scaling in Si technology has exaggerated all three kinds of failures, years of manufacturing research accompanied with conventional architecture and circuit-level reliability solutions have made 2D Si a robust technology [36]. On the contrary, for 3D integration and emerging devices, the situation is worse because of the early stages of development and a higher rate of infant and random period errors [52]. Moreover, increased levels of operating temperature, electromigration, current density, and thermal variation in 3D technologies speed up the aging failures [27, 29, 34, 43, 54, 56]. Extensive research on both the manufacturing and architecture fronts are required to move innovation forward to create large-scale chips. However, aggressive manufacturing research usually will not be done unless a product is marketed, and products cannot be developed profitably because of the challenges in the manufacturing and lifetime, leading to a vicious cycle. Hence, to break this causality dilemma, we present R2D3 as an architectural innovation to improve reliability concerns of the emerging M3D technology.

A naïve reliability solution is to incorporate system-level redundancy, such as dual/triple modular redundancy, which is expensive, inefficient, and only applicable to high-end systems. Prior works leverage the redundant nature of multi-core systems that allow low-cost repair by disabling defective cores using hardware or software mechanisms, which is suitable for standard technologies like 2D silicon technology [5, 11, 14, 22, 37, 47]. However, adopting core-level mechanisms for high-fault-rate technologies can cause multiple device failures to discard many cores at once, oftentimes with the majority of the components of the faulty core still functional, which could have been easily salvaged. Therefore, these solutions do not scale well with high fault rates. This motivates a rethinking of the architectural fabric from the ground up, with dynamic adaptivity and configurability as primary requirements. To be effective, the fault isolation has to be at a granularity finer than core level. Although there is a plethora of work to address reliability concerns, no work addresses all four features of reliability concurrently. Hence, we categorize previous work into fault detection and diagnosis, repair, or lifetime management solutions as shown in Table 3. The issues with previous solutions can be summarized as follows:

First, some solutions lack essential features to be considered practical. For instance, previous reconfigurable architectures such as StageNet [20], Core Cannibal architecture [45], 3DFAR [7], and Viper [42] lack fault detection and diagnosis at a finer granularity, which makes them incomplete. Second, studies show that elevated temperatures and longer heat dissipation paths in 3D ICs lead to rapid aging and higher fault rates [27, 54], and this calls for an end-to-end solution that can control aggressive aging and repair the system upon a fault. Third is the high-overhead Frankenstein issue: while some prior work may seem to incur a lower overhead in a particular category 
in Table 3, they do not provide all the four features of reliability. Even if we ignore compatibility issues and combine multiple solutions together, in a Frankenstein method, the system will incur a high performance penalty and area overhead. For instance, StageNet [20], core cannibal architecture [45], 3DFAR [7], and viper [42] lack fault detection and diagnosis at a fine granularity, which is essential to their repair system. This introduces additional overhead on top of what they propose as fault detection and diagnosis at a fine granularity, which can be expensive [6, 36, 46]. Moreover, the mentioned solutions miss out on considering usage and heat, leading to non-uniform aging and, hence, lower lifetime and higher faults.

Low-yield and high-fault-rate technologies call for the incorporation of an end-to-end solution that provides all the four pillars at a low overhead, motivating the need for a unified solution at a fine granularity. To resolve these issues, our solution proposes to utilize the third dimension, to provide a unified reliability solution with concurrent fault detection, diagnosis, repair, and graceful $V_{t h}$ degradation, compared to the limited and specialized approaches mentioned above. With this, R2D3 delivers a substantially reduced-overhead solution, as shown in Table 3, when compared to prior work.

\subsection{Opportunities Born from Challenges}

While M3D technology is associated with certain challenges such as low yield and high fault rate, it also provides opportunities like leftovers and fast 3D reconfiguration fabric to resolve the challenges. Leftovers are salvaged redundant pipeline stages that are functional but cannot be used to form a complete core. There are two scenarios in which a leftover can be available:

(1) Idle functional pipeline stages of faulty cores that cannot form a complete core (orange stages in Figure 2)

(2) Pipeline stages in other working cores that are powered off temporarily because of a low utilization workload or power and temperature constraints

Furthermore, the growing gap between the number of cores that can be placed on a chip and those that can be powered on simultaneously, referred to as the Many-Core Power Wall [28], increases the number of available leftovers. These leftovers can be used to provide the redundancy that is required to support not only treatment but also prevention. This forms the foundation of our unified reliability solution.

Fast 3D reconfiguration fabric: Monolithic 3D fabric allows the placement of identical resources within short vertical distances from one another, providing a low overhead and fast interconnect network using MIVs as shown in Figure 3. Cross-layer interconnect switches do not require any buffering, thus greatly simplifying their design and control requirements. Moreover, it is possible to use switches to connect pipeline stages on both forward and backward paths. Note that prior reconfigurable solutions like [20] suffer from performance and complexity impacts introduced by buffered switches. However, since propagation delays on vertical MIVs are minimal (approximately two orders of magnitude faster than in conventional 2D layouts) due to the much shorter lengths to be traversed, we can avoid buffering by accommodating a small increase in clock cycle length $(<8.2 \%)$. This can be crucial for adopting our solution for more complicated architectures. To evaluate, we analyzed the propagation delays for two distinct layouts of a four-core OpenSPARC T1 processor. The first is a 2D layout with the four cores in a $2 \times 2$ formation with switches placed at the center of the formation, and the second is a 3D layout with the four cores stacked above each other, similar to the schematic of Figure 2. For a $45 \mathrm{~nm}$ IBM technology, the worst-case propagation delay for signals going from the output of one stage, through an interconnect switch, and to the input of the next stage for the $2 \mathrm{D}$ layout is $950 \mathrm{ps}$, compared to only $50 \mathrm{ps}$ for the $3 \mathrm{D}$ layout, a $95 \%$ reduction. This vast difference is due to the much shorter distances that 


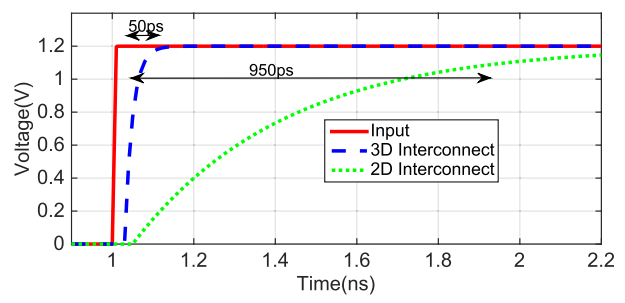

Fig. 3. Propagation delays through inter-stage interconnects for a four-core planar layout vs. a 3D stacked layout. The large delay for the planar design is mostly due to the much longer wires required to connect corresponding units.

must be traversed to reach a corresponding unit in the three-dimensional solution. Based on these findings, our interconnect switch designs and checkers only add an $8.2 \%$ frequency overhead.

\subsection{Intuition on Lifetime Management}

In this section, we demonstrate the effect of temperature profiles on aging in $3 \mathrm{D}$ circuits and provide intuition by an example of uniform $V_{t h}$ degradation due to NBTI via adaptive and dynamic reconfiguration policies. In order to account for static and dynamic NBTI degradation, we use the model [57] used to compute the change in threshold voltage caused by NBTI degradation for the stress and recovery phases as follows:

$$
\begin{aligned}
& \text { Stress : } \Delta V_{t h}=\sqrt{K_{v}^{2}\left(t-t_{0}\right)^{1 / 2}+\Delta V_{t h 0^{2}}} \\
& \text { Recovery }: \Delta V_{t h}=V_{t h}\left[1-\sqrt{\eta\left(t-t_{0}\right) / t}\right]
\end{aligned}
$$

where $\Delta V_{t h}$ is the change in threshold voltage caused by NBTI stress and recovery phases, $t_{0}$ is the starting time of the stress or recovery phase, $\Delta V_{t h 0}$ is the threshold voltage change starting each stress or recovery phase at $t_{0}$, and $K_{v}$ is a constant that combines all the technological parameters [57]. NBTI degradation is highly impacted by voltage $\left(V_{g s}\right)$ and temperature $(T)$, higher values of which increase the aging rate. Different heat dissipation paths in 3D ICs lead to nonuniform temperature across its physical layers. Layers that are farther from the heat-sink have higher temperatures and temperature instability, and consequently accelerated $V_{t h}$ degradation. Figure 4(a) shows the $V_{t h}$ degradation of different layers in a four-core processor in 3D over 100 seconds, where each core is placed in a layer. All cores are switched on and off for consecutive periods of 10 seconds, thus making the activity factor of each core equal to 0.5 . In this setting, the difference between the temperature of the top and bottom cores is $28^{\circ} \mathrm{C}$.

For processors with more layers, the difference in temperature between the coolest and hottest cores becomes greater, resulting in a larger gap between their $V_{t h}$ degradation ratios. Increasing the number of cores to eight elevates the temperature difference of the coolest and hottest cores to $51^{\circ} \mathrm{C}$, which would cause an order of magnitude difference in the NBTI degradation rates. The increase in supply voltage in response to elevated threshold voltage causes performance degradation and higher power consumption. This leads to higher temperature and faster aging not only in the hottest core but also in cores in the adjacent layers. Hence, controlling and balancing the degradation of a multi-core processor in 3D fabric are more critical in comparison to 2D systems.

Figure 4(b) illustrates the $V_{t h}$ degradation of the cores at varying activity factors. For a particular activity factor, due to temperature variations among layers, each core experiences a different $V_{t h}$ degradation. However, the key takeaway and motivation for this work is from the observation 


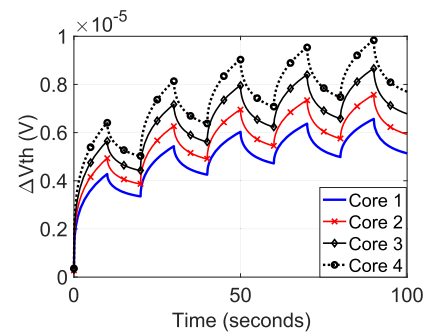

(a)

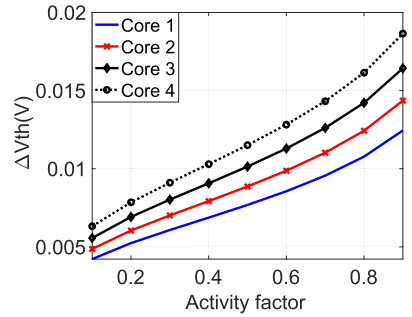

(b)

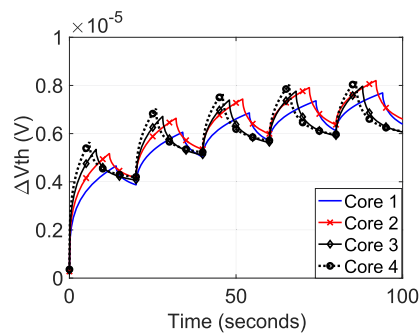

(c)

Fig. 4. $V_{t h}$ degradation of cores in a four-core processor in 3D for (a) equal on and off periods (identical activity factors). All cores are on and off for consecutive periods of 10 seconds. (b) Varying activity factors for the four cores. (c) Unequal on and off periods (nonidentical activity factors). The difference between the temperature of the coldest and hottest cores is $28^{\circ} \mathrm{C}$.

that we need to reconfigure pipeline stages to ensure they run at varying activity factors to yield a uniform NBTI-based $V_{t h}$ degradation across all cores on different layers. For instance, in order to achieve uniform $\Delta V_{t h}=0.008 \mathrm{~V}$ across all cores, the activity factor of each core should be $\alpha_{1}=0.53$, $\alpha_{2}=0.41, \alpha_{3}=0.30$, and $\alpha_{4}=0.21$, respectively, where $\alpha_{1}$ is the activity factor of the core closest to the heat sink, while $\alpha_{4}$ is that of the core farthest away in a four-core system. By adopting these relatively balanced activity factors, we obtain a more uniform $V_{t h}$ degradation across the cores, as illustrated in Figure 4(c). Drawing inspiration from this idea that balances aging on the core level, we tune it to the pipeline stage level for a fine-grained policy.

\section{PROPOSED FRAMEWORK}

This section describes our baseline architecture that provides fault repair, along with our proposed fault detection and diagnosis mechanism, and two reconfiguration policies to combat aging.

\subsection{Architecture}

R2D3 leverages the natural redundancy available in parallel systems to provide robustness against permanent transistor failures. Unlike classic architectures that execute instructions on paths fixed at design time, it relies on inter-stage crossbar switches to form logical pipelines dynamically. Figure 5 shows the high-level components of R2D3, where corresponding pipeline stages are stacked vertically and vertical buses that function as crossbars are inserted between consecutive stages. By replacing the direct connections at each pipeline stage boundary with interconnect switches, we create a network of resources in which each pipeline stage is connected to all instances of the subsequent stage. At the layer closest to the heat-sink, we insert the reconfiguration controller and detection circuitry, which consists of two comparators between subsequent stages, for all layers. The reconfiguration controller can dynamically configure the interconnect to route instructions through functional hardware and detour around failed units, as shown in Figure 2.

Interconnect switch design: For the interconnect switch design, we adopted a bus style presented in [7], where vertical links run across the entire height of the design, and each layer uses a set of multiplexers to select its inputs from the prior stage on the same layer or the prior stage from other layers using the vertical bus lines. The advantage of this solution is that only unidirectional MIVs are required, since signals are switched directly at their destination layer.

To minimize the performance loss from inter-stage communications, we use multiplexer-based full crossbar switches. The MUX-based crossbar has a fixed channel width and, as a result, the 


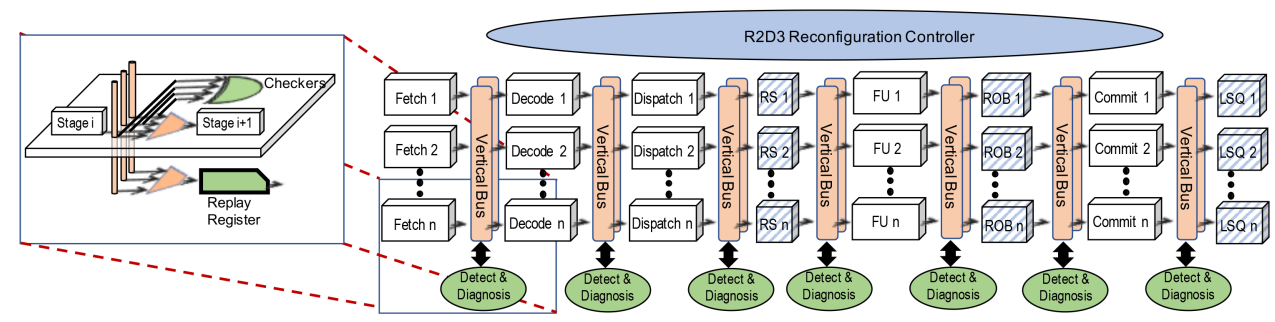

Fig. 5. Schematic showing components of R2D3, where pipeline stages are stacked vertically and vertical buses that function as crossbars are inserted between consecutive stages. At the end of each epoch, detection circuitry can access the inputs and outputs of all layers to check the output, and the R2D3 Reconfiguration Controller manages the components.

delay of transferring an instruction from one stage to the next can occur with a small frequency overhead within the same clock cycle when implemented in 3D [7].

A novel framework rather than a new architecture: Previous reconfigurable architectures such as StageNet rely on buffered switches with limited bandwidth between consecutive stages These switches in each pipeline stage boundary complicate the design as different instructions may take a different number of clock cycles to reach the destination and a more complicated mechanism and control infrastructure for ensuring correct execution. More importantly, these extra buffers between stages introduce performance overhead in terms of IPC as well. Consequently, writers in the StageNet paper had to change and redesign the structure of the five-stage in-order pipeline to create a four-stage processor and reduce the number of signals and amount of data between each of the two stages. The performance overhead and cost of redesigning the whole processor make the adaptation of these architectures for more complicated architectures such as out-of-order cores or those with more stages too expensive. In the case of R2D3, since propagation delays on vertical MIVs are minimal (approximately two orders of magnitude faster than in conventional 2D layouts) due to the much shorter lengths to be traversed, we can avoid buffering by accommodating a small increase in clock cycle length. There are now buffers and added pipeline registers between two consecutive stages and, hence, no change needed to modify the architecture of the processor. This is one of the factors that distinguishes R2D3 from previous solutions; previous solutions in fact are proposing a new architecture with its specific limitations and applications, but this work tries to introduce a new framework that can be adopted for any architecture when used in emerging monolithic 3D fabric.

Out-of-order architectures: As mentioned in the previous part, R2D3 is a new framework that can be adopted for any architecture that consists of elements that can be decoupled. R2D3 decouples these stages but does not change the baseline architecture using crossbars (vertical bus interconnect) that are combinational logic. Hence, it does not change the basic architecture, nor does it have any effect on the instruction flow within a pipeline. As the pipeline-stage boundaries are very distinct in any modern processor, it is fairly straightforward to adopt R2D3 for any modern in-order or out-of-order cores. On the other hand, OOO cores contain more buffers and structures with more memory elements such as Re-Ordering Buffer (ROB), Reservation Station (RS), and Load-Store Queue (LSQ), as shown in Figure 5. These elements can be easily decoupled using our vertical bus interconnect, but switching between them can be challenging as the state will be lost if swapping out one of them. This issue can happen in the case of any in-order core for structures such as register files. To resolve this issue, we introduce a mechanism called the warm-up. To accomplish this, a few cycles before $T_{\text {sched }}$, we warm up the next stage to be used by duplicating operations and forwarding all the necessary data using the vertical bus interconnect. Thus, at 


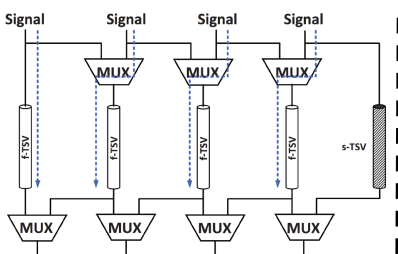

(a)

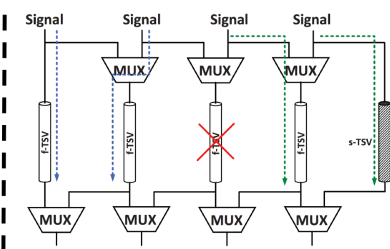

(b)

Fig. 6. A conceptual figure on how spare MIV methods work. (a) Operation when all f-MIVs are fault-free. (b) Operation when the third f-MIV is faulty.

the time of reconfiguration, the next stage will be substituted in seamlessly and the only action needed would be to shut off the existing working module. So the reconfiguration does not entail any additional performance overhead as it does not halt the program execution for swapping the stressed resource with its substitute.

\subsection{Vertical Connections Reliability}

Low MIV and TSV yield and reliability are serious problems, as the failure of a single MIV may cause unpredictable effects that could eventually lead to system failure. Yield and reliability improvements are usually achieved by different forms of redundancy. MIV yield improvement using spares has been widely investigated, and many diagnosis and repair mechanisms have been proposed in this regard $[26,59,60]$. The addition of spare MIVs is an effective method for yield and reliability enhancement, but this approach results in hardware cost and delay overhead. In the double MIV technique, each faulty MIV (f-MIV) is paired with an additional spare MIV (s-MIV) to enhance yield and reliability [26]. In the fault-free scenario, a signal is transferred through the two MIVs simultaneously. Once an f-MIV becomes faulty, there is still an sMIV to pass the signal. Since the two MIVs are used to pass the same signal, no additional control circuits are required. However, due to the significant area overhead induced by s-MIVs, this technique is impractical.

To reduce area overhead, the "shared s-MIV" technique has been proposed. In this technique, a set of f-MIVs is partitioned into several groups, and single or multiple s-MIV(s) are subsequently assigned to each group. By inserting MUXes and carefully designing the reconfigurable routing paths, the s-MIV(s) can be used to pass signal(s) in the presence of defective f-MIVs. Figure 6 illustrates an example of a shared s-MIV structure. When all the f-MIVs are fault-free, the signals are transferred by them in Figure 6(a). Once an f-MIV fails, the signal corresponding to the faulty one has to be shifted, which causes all signals between the faulty MIV and the sMIV to be shifted, as shown in Figure 6(b). Although this method reduces the area overhead compared to the doubleMIV technique, additional delay is introduced due to signal re-routing and the extra components.

\subsection{Cluster Size and Number of Design Layers}

There are contrasting goals in determining the ideal number of layers in an R2D3 design: on one hand, the more the layers, the more spare units are available, and thus the stronger the robustness of the solution. On the other hand, a high number of design layers may be impractical and may negatively affect the latency required for traversing the vertical dimension of the design to reach a spare unit. In this case, a large many-core system would need to be logically divided into smaller R2D3 clusters. Each such cluster would offer full connectivity within itself. Increasing the cluster size will improve reliability as there will be more accessible resources and a reconfiguration option in the case of fault. In order to ascertain the right number of layers that can be efficiently stacked together to form a cluster, we conducted reliability and overhead experiments for different cluster 


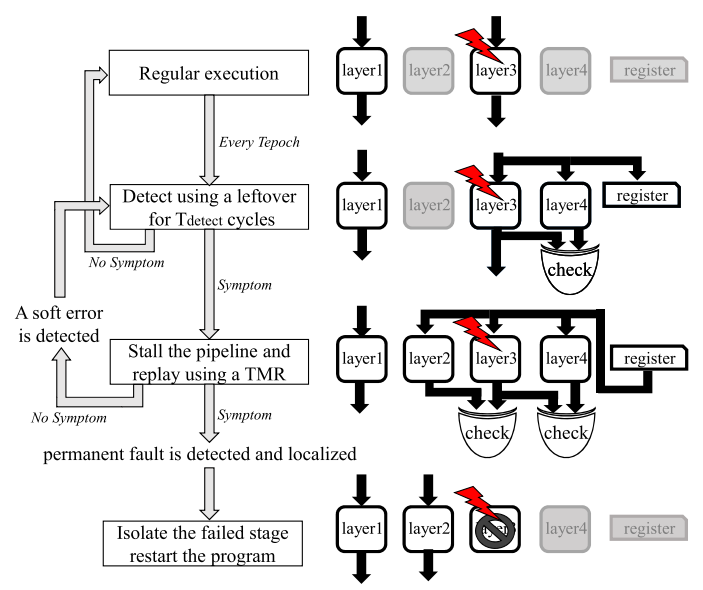

Fig. 7. R2D3 fault detection, diagnosis, and repair mechanism in a flowchart (left) and the hardware configuration of the pipeline stages in a four-layer design in each phase (right).

sizes, which is investigated in detail in the experimental results section. We study this trade-off in the experimental evaluation section.

\subsection{Fault Detection and Diagnosis}

This subsection introduces our framework to detect faults, distinguish between a transient and permanent fault, and localize it to a single pipeline stage. We take advantage of R2D3's fast reconfiguration fabric to propose a low-cost and reliable design. The close proximity of vertical stages further helps reduce the overhead associated with the comparison of the output, efficiently locating the failure. We consider independent instruction streams (from different threads/tasks) executing on each logical pipeline that do not have the same resource utilization (occupancy) profile. This provides us an opportunity to re-execute instructions on leftovers located in the proximity of the design under test (DUT) stage.

Our detection and diagnosis scheme utilizes the above concept and does not require any additional redundancy. There is near-zero performance penalty with the concurrent utilization of idle resources. The scheme runs online; i.e. it functions in the background during normal application execution and is completely transparent to software. We only consider faults in the core datapath and assume a single-fault model; i.e., at any point of time, a single stage can become faulty.

R2D3's detection and diagnosis feature does the following:

(1) Detection: Creates coarse-grained computational epochs that involve the use of leftovers to execute identical instructions as those running on the DUT stage

(2) Diagnosis: Distinguishes between a transient fault and a permanent fault and determines which stage is faulty using a single replay for one clock cycle

Fault detection: Each epoch takes $T_{\text {epoch }}$ cycles and determines how often a particular stage is tested. At the end of each epoch, we exploit the leftovers to verify the functional integrity of each stage of the logical pipelines for $T_{\text {test }}$ cycles by executing the same instructions as those running on the DUT stage. If no fault is detected during the online testing, indicating the underlying hardware is known to be free of silicon defects, the epoch's computation is ended.

As shown in Figure 7, the results are compared using simple inter-stage checkers at the output of the pipeline stages in order to be able to assess the faulty behavior of the involved blocks. Having a fast 3D reconfiguration fabric, the output and input of each pipeline stage can be controlled 
and monitored at the layer with the crossbar switches. If the input of two similar stages in two different layers is the same, the output of the two should be identical too. We do not need additional connections as the I/Os of each pipeline stage are available within the R2D3 structure in any layer. Regardless of the layer the checkers are in, they can access the I/Os of all stages through R2D3's vertical buses.

Eliminating the performance penalty caused by halting the pipeline for testing, R2D3 provides a unique opportunity to enhance fault detection. First, it is now possible to evaluate the underlying hardware for a longer period (higher $T_{\text {test }}$ ), allowing rigorous testing operations. Second, it is possible to reduce the epoch duration, testing each stage more frequently (smaller $T_{\text {epoch }}$ ). Hence, the delay for fault detection can be reduced and the performance penalty caused by using the faulty hardware is minimized. On the other hand, using the leftovers for fault detection adds power overhead. There is a trade-off between the test duration/fault coverage ratio and the added power overhead. As shown in Figure 7, we also copy the inputs of the two stages that have been tested into our register for one clock cycle; hence, in the case of a fault we are able to replay them to diagnose the fault. For fault detection, before starting to run the instructions on the second stage, we warm up the next stage to be used by forwarding all the necessary data containing the state of the registers, branch prediction data, and register file using the same crossbar. Then, when everything is set on the second stage, we start the parallel re-execution. Thus, at the time of reconfiguration, the next stage will be substituted in seamlessly.

Fault diagnosis: After a symptom is detected, the fault needs to be diagnosed, i.e., distinguish between transient and permanent hardware faults, and, in the case of a permanent fault, identify the faulty component to initiate system repair. Then the diagnosis algorithm of R2D3, as shown in Figure 7, achieves this with the help of another leftover and the ability to replay an execution using the inputs of the previous cycle, which caused the symptoms, stored in registers. R2D3 uses a simple step to distinguish between transient faults and permanent faults and localize it: it halts the pipeline for one clock cycle and replays the symptom-generating instruction on the two symptomgenerating stages and a new known third good stage using Triple Modular Redundancy (TMR). If the symptom does not recur, a transient fault was detected and the execution continues after having stalled for only one clock cycle. If the symptom recurs on re-execution, i.e., a hard fault, the third stage is used to vote and determine the faulty one. Hence, localizing a permanent fault at a pipeline-stage granularity facilitates fine-grained repair and reconfiguration.

\subsection{Repair}

By adaptively routing around failed stages, we can salvage working units and repair the system. When a fault occurs, the victim unit (i.e., a pipeline stage) is isolated and the reconfiguration procedure is initiated, constructing logical pipelines based on the latest failure map. Since leftovers can be on any layer, the executing pipeline may comprise elements from various vertical layers. In Figure 2, four faults have disabled units on different vertical layers. R2D3 can build two complete pipelines dynamically, as shown by the red and green wideband lines, while a core-level solution would have only one functioning core. Once reconfigured, we re-execute the task from a checkpoint or the beginning.

\subsection{R2D3 Aging-Aware Policy}

As discussed earlier, while previous reconfigurable solutions provide fault tolerance, they miss out on the opportunity to leverage this reconfigurabilty to decelerate aging. Hence, we propose a reconfiguration policy that is aging aware and provides all treatment methods. In prior reconfigurationbased reliability solutions $[7,20]$ like Static 3D, when a new failure appears in a working stage, a static reconfiguration is applied to create logical cores by randomly choosing available functional 
stages. Initially, the new faulty pipeline is suspended and all processes in execution are swapped out of context by the operating system. Then, the reconfiguration routine computes the number of working pipelines that can be created with the current failure map and programs the interconnect switches accordingly. The operating system then takes over to reconfigure all the processes in execution, based on the remaining functional pipelines. However, once the reconfiguration is complete and the logical cores have been set up, no further modifications or changes are made unless another fault is detected. Hence, the main difference in comparison to the NoRecon case is that the system is repaired using a static reconfiguration policy. However, upon a fault, this scheme uses fixed resources across the 3D stack that are chosen at reconfiguration time, while the leftover pipeline stages are shut down. Consequently, the used resources are worn out at a higher rate and experience a larger degradation in threshold voltage, considerably reducing the system's lifetime. Our solution utilizes leftover pipeline stages to provide an opportunity for the resource under stress to partially recover $V_{t h}$. This enables R2D3 to effectively control and evenly distribute wearout among all working stages. Based on this, we propose two dynamic reconfiguration methods, R2D3-Lite and R2D3, that aim to reduce the $V_{t h}$ degradation (recoverable when not under stress) and minimize its effect on NBTI-based aging.

R2D3-Lite: We take advantage of the opportunity that we can replace any working stage with a leftover from another layer. Initially, we reassign leftovers in a round-robin fashion after a certain reconfiguration time period, $T_{\text {sched }}$. In this scheme, the policy assigns equal probabilities to choose pipeline stages to create logical cores. For example, in the case that three non-fault execution stages are available in the system and only two of them are being utilized, the probability to use any of them would be $67 \%$. Therefore, the policy switches to the third redundant stage after every $T_{\text {sched }}$ cycle. Not only does this scheme balance the usage of resources in the processors, but also it gives the units a chance to be unstressed and partially recover their $V_{t h}$ degradation. This policy equalizes the usage of all the pipeline stages, which does not necessarily balance wearout, as the cores farther away from the heat sink will have higher temperatures.

R2D3: To expand on R2D3-Lite, R2D3 was specifically designed to address the characteristics of the 3D system and provide uniform aging. R2D3 assigns an activity index, $A_{i}$, to each stage based on its temperature profile and location in the stack; i.e., if the activity index of a stage is lower, then it is more prone to hot spots and degradation. Using the pre-calculated data obtained based on temperature patterns and $\Delta V_{t h}$ as shown in Figure 4(b), the policy updates these indices during execution and adaptively utilizes each pipeline stage based on its activity index while reconfiguring. For instance, to obtain a uniform $\Delta V_{t h}=0.008 \mathrm{~V}$, the activity factor of each stage in different cores would be $\alpha_{1}=0.53, \alpha_{2}=0.41, \alpha_{3}=0.30$, and $\alpha_{4}=0.21$, respectively, where $\alpha_{1}$ is the activity factor of the stage in the core closest to the heat sink, while $\alpha_{4}$ is that of the core farthest away in a four-layer system. To balance $\Delta V_{t h}$, the relative activity factor of the cores needs to follow the predicted values. The policy is modeled to favor stages that are less likely to wear out and heat up in the near future by increasing the usage probabilities of cooler stages. Going back to the example in Figure 2 where EXE1, EXE2, and EXE4 are functional, the relative utilization of EXE2 should be $\alpha_{2} /\left(\alpha_{1}+\alpha_{2}+\alpha_{4}\right)$. This is the balanced relative utilization for the case in which only one running core is needed, and if $n_{\text {workload }}$ is the number of cores that are needed to be run, the activity factor of EXE2 also needs to be multiplied by that ratio. This would give us the activity index of EXE2, $A_{E X E 2}$, which is the relative activity that is expected from this stage to run the workload and balance usage as demonstrated by the following:

$$
A_{i}=\frac{\alpha_{i}}{\sum_{j=1}^{n_{\text {live }}} \alpha_{j}} \cdot n_{\text {workload }}
$$


where $\alpha_{i}$ is the predicted activity factor for pipeline stage $i, n_{\text {live }}$ is the number of pipeline stages of the same type that are functional and available, and $n_{\text {workload }}$ is the number of cores required to run the workload $\left(\leq n_{\text {live }}\right)$. The activity indices are calculated every period of calibration window $\left(T_{c a l}\right)$ and each of the available resources is scheduled based on their activity index within $T_{\text {cal }}$ as follows:

$$
T_{\text {sched }, i}=A_{i} \cdot T_{\text {cal }},
$$

where $T_{\text {sched, } i}$ is the time period over which pipeline stage $i$ is scheduled to be functionally active. These activity factors can be determined either offline based on the steady state temperature of cores for typical workloads (and therefore implicitly based on the location of cores) or at runtime based on the temperature and wearout $\left(\Delta V_{t h}\right)$ history. In this work, we used predicted activity factors based on the steady state temperature simulations that are described in Section 4.

A final advantage is that both of these reconfiguration policies do not entail any additional performance overhead as they do not halt the program execution for swapping the stressed resource with its substitute. To accomplish this, a few cycles before $T_{\text {sched }}$, we warm up the next stage to be used by duplicating operations and forwarding all the necessary data. Thus, at the time of reconfiguration, the next stage will be substituted in seamlessly and the only action needed would be to shut off the existing working module.

\subsection{Assumptions and Limitations}

3D vs. 2D: We focus on 3D architectures in which elevated temperatures and longer heat dissipation paths due to stacking computational units lead to non-uniform temperature and delay variations across the layers. Although 3D integration has not yet been realized for commercial fabrication, it is one of the most promising emerging solutions to improve transistor density [25]. However, the practicality of its implementation is out of the scope of this article. Nonetheless, this framework can be built upon all previous 2D and 3D fault-tolerant reconfigurable solutions, enhancing their performance and lifetime.

Addressing soft faults: Our approach in its current form is that it can only be used to detect and correct transient faults that happen during the test time $\left(T_{\text {test }}\right)$. Zhang et al. [63] characterize soft error vulnerabilities across the stacked layers under 3D integration technology and show that the outer dies can shield more than $90 \%$ of particle strikes for the inner dies, which leads to an order of magnitude reduction in soft error rates compared to a similar 2D chip. Hence, for the outer layers more prone to particle strike, our method can assign a longer testing time $\left(T_{\text {test }}\right)$ to increase the probability of catching the generated soft error rates. Moreover, critical instructions can be mapped to the more robust inner layers.

Caches and system-level operation: The L0 caches also contain a $8 \times 8$ crossbar (not shown in Figure 2) and have a similar structure to the design in [39]. Hence, caches and TLBs are separated from the stages' logic and considers to be input to the stages, which is the case for most modern processors and enables efficient cache pooling for better resource management [39]. This allows seamless effort and no additional overhead from the memory side in the case of reconfiguration or running two stages in lockstep for fault detection. Additionally, the cache organization must be set-associative to accommodate both speculative and non-speculative states. Moreover, we assume that faults in local caches are handled with a finer granularity approach like Error-Correcting Codes (ECCs).

Requirements of architecture: The approach of disabling defective functional units in a solution such as R2D3 requires multiple units of each class. Otherwise, a single defect in a critical 
non-replicated unit such as central control logic could disable the processor. As such, the stage comparison logic needs to be implemented in TMR logic. The area and frequency overhead may increase based on how tightly the baseline architecture is pipelined. However, considering that modern processors usually use Dynamic Voltage-Frequency Scaling (DVFS) and rarely function at the full frequency, R2D3 frequency overhead really has a small effect over the lifetime of the processor.

Crossbar reliability: Using centralized switches could result in a single point of failure. We leverage a bus-style architecture [7] that is equally distributed between all layers. Each layer contains the logic for routing the appropriate instructions for that particular layer. In other words, each layer uses a set of multiplexers to select its inputs among one of the vertical bus lines or the prior stage on the same layer and avoiding the single point of failure. As described in [7], this interconnect consists of a number of identical busses, and each stage and layer can access any of the busses. For instance, for an eight-layer design, it has four identical busses, which gives the network an inherent redundancy. The Mux that chooses the input of the stage is considered as the part of the stage, so a failure in it would be treated as a failure in the stage, which entails reconfiguration and isolation of the entire stage in that layer.

Effect of technology scaling: Technology scaling can play two roles that need to be considered for adopting R2D3. First is how 3D design roles and vertical connections would scale. If they scale at the same factor, the total frequency and area overhead would remain the same, but if they lag, then the total cost of deployment can increase. This can be difficult to predict as 3D technology is currently in the development phase. The current research-level data shows that it is really difficult to scale these vertical connections beyond a point. This will increase the cost of R2D3 deployment in more aggressive technologies. The other important factor that needs to be considered is the effect of scaling on power, temperature, and hence reliability, especially in terms of 3D circuits. Smaller technology nodes have more fragile devices and interconnect and higher levels of power density and temperature and, hence, suffer from more aging and failures. Our analysis shows that the effect of R2D3 in performance improvement increases as the number of faults in systems increases. so in this condition R2D3 can add more value by the cost it adds to the systems.

\section{EXPERIMENTAL METHODOLOGY}

This section illustrates the main steps of the proposed reliability evaluation mechanisms to address technology failures and NBTI-based wearout failures. For fault simulation, we use the Synopsys TetraMAX ATPG tool to generate test patterns for the synthesized netlist. In our studies, we explored the stuck-at fault model, which is the industry standard model for test pattern generation. It assumes that a circuit defect behaves as a node stuck at 0 or 1 . Our fault model injects permanent transistor failures into any design component and any layer, proportionally to the area of the unit. Once a pipeline unit is hit by a fault, we disable the entire unit and trigger a dynamic reconfiguration. We assume that faults in local caches are handled with ECCs. Moreover, if a fault hits an interconnect switch, we disable the unit connected to the output of that switch. If a fault hits the pipeline's control logic, we disable the entire pipeline. We assume that MIVs are implemented reliably as discussed in Section 3.7; we accounted for 1 spare MIV forr every 100, based on the recommendation in [26].

A brief overview of our approach is shown in Figure 8. We start by considering a given hierarchical description of the design. This description can be provided in any hardware description language such as VHDL or Verilog. In addition, technology parameters are derived based on the technology node in which the design is to be fabricated. In this article, we evaluated Silicon because process parameters and aging models are easier to acquire. R2D3 can be implemented on 


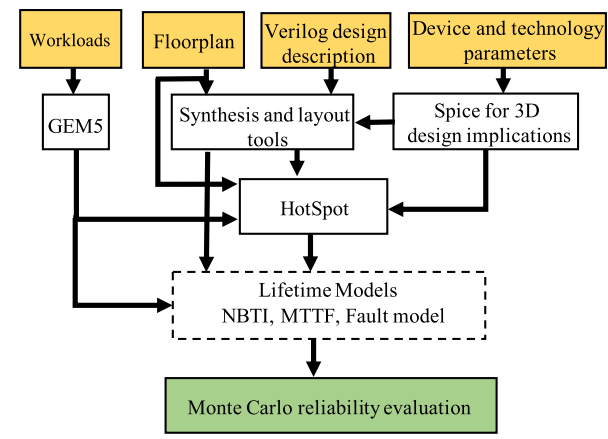

Fig. 8. Flow chart of the proposed reliability evaluation methodology for NBTI failure mechanism.

top of any emerging device that supports 3D integration, but due to lack of definite wearout and technology models for these technologies, we push the evaluation of R2D3 on these systems to future works.

We synthesized our design on a commercial $45 \mathrm{~nm}$ SOI process technology with the Synposys Design Compiler and performed place-and-route of the design using Cadence Innovus. To create the M3D layout of the design, we follow the specifications and design rules recommended by Dae et al. in [30] for monolithic 3D integration and evaluate power and timing through SPICE simulations. The resulting layout represents the block-level floorplan, in which each block is further divided into individual structures or sub-blocks based on the initial structure of the design. This hierarchical design helps us obtain the layout, location, and aspect ratio of each sub-block. The physical design and the physical parameters and power consumption estimates generated from Synopsys signoff tools are collected.

The floorplan and power estimates are then fed into HotSpot [61]. HotSpot is an accurate and fast thermal model based on an equivalent circuit of thermal resistance and capacitance that corresponds to microarchitectural blocks. We use HotSpot Version 6.0 [61] for thermal modeling. Using the $3 \mathrm{D}$ capability available in grid mode, the proposed floorplan of the system is incorporated into the analysis. Also, we use the default characteristics provided by the tool for our die package, as these represent a modern CPU package. The output of the HotSpot simulation is a list with temperatures of each sub-block. The temperature of sub-blocks along with the circuit netlists generated using the Cadence tools are utilized to perform sub-block-level SPICE simulations. These simulations provide us with the transistor operating parameters necessary to be plugged into the equations modeling the wearout mechanisms. It is important to note that the depth of design hierarchy utilized directly impacts the computational runtime; i.e., it increases with larger designs.

The core of the proposed methodology relies on a Monte Carlo simulation algorithm implemented and run in MATLAB. Our technique is executed at the sub-block level, where the base unit is a transistor. Moreover, at each calibration window, based on the fault pattern and predicted activity factors of the available modules, we calculate the activity index of each core and schedule based on (Equation (3)) and (Equation (4)). As NBTI degradation is independent of frequency and stress and recovery phases $[23,57]$, we choose our reconfiguration frequency to optimize power and performance overhead and fault coverage. We set our calibration window for R2D3 equal to 5 $\mathrm{ms}$ (5M cycles), which is based on the studies that are presented in Section 5.3.2. During this calibration window, a few cycles before every $T_{\text {sched }}$, we start the process of handing over from one functional stage to another as described in Section 3. Furthermore, to attain statistical confidence, we repeat each experiment on faulty processors 10,000 times, using varying random seeds. 
Table 1. The Simulation Parameters for R2D3

\begin{tabular}{|c|c|c|}
\hline Module & In-order Core Parameters & OOO Core Parameters \\
\hline Core & $\begin{array}{l}\text { SPARC V9 core, 5-stage pipeline @ } 1.0 \mathrm{GHz} \text {, 3-cycle } \\
\text { pipelined integer and floating-point FUs }\end{array}$ & $\begin{array}{l}\text { RV64 BOOM, 10-stage pipeline @ 1.0 GHz, 4-stage } \\
\text { pipelined integer ALU, pipelined floating-point }\end{array}$ \\
\hline ache & $\begin{array}{l}8 \mathrm{kB}, 4 \text {-way set-associative, private cache with } 8 \text { MSHRs } \\
\text { and } 64 \mathrm{~B} \text { block size with stride prefetcher }\end{array}$ & $\begin{array}{l}16 \mathrm{kB} \text {, 4-way set-associative, private cache with } \\
4 \text { MSHRs }\end{array}$ \\
\hline L2 D-Cache & $\begin{array}{l}64 \mathrm{kB}, 4 \text {-way set-associative, shared cache with } 8 \text { MSHRs } \\
\text { and 64B block size }\end{array}$ & $1 \mathrm{MB}, 8$-way set-associative, shared cache with $8 \mathrm{MSHRs}$ \\
\hline $\mathrm{I}-\mathrm{C}$ & $\begin{array}{l}4 \mathrm{kB}, 4 \text {-way set-associative, private cache with } 4 \text { MSHRs } \\
\text { and } 64 \mathrm{~B} \text { block size }\end{array}$ & $\begin{array}{l}16 \mathrm{kB}, 4 \text {-way set-associative, private cache with } \\
4 \mathrm{MSHRs}\end{array}$ \\
\hline $\begin{array}{l}\text { Main } \\
\text { Memory }\end{array}$ & 4-channel DDR4-2400 x64 @ 18.8 GB/s per channel & 4-channel DDR4-2400 x64@18.8 GB/s per channel \\
\hline
\end{tabular}

For our out-of-order core, we used the RISC-V BOOM core [9] using the Chipyard tool set [4], which includes the infrastructure to set up the environment, build, and run the BOOM core on a $\mathrm{C}++$ emulator. Chipyard also provides supported flows for pushing a BOOM-based SoC through the ASIC flow. The gem5 simulator [8] and BOOM C++ emulator were used to simulate the performance of the in-order cores. The parameters used for simulating the proposed architecture are shown in Table 1. We use 13 workloads of the PARSEC3.0 benchmark as well as three popular kernels, general matrix-matrix multiplication (GEMM), general matrix-vector multiplication (GEMV), and fast Fourier transform (FFT). FFT is widely used in communication and visual processing systems. GEMM and GEMV are ubiquitous kernels in machine learning, scientific workloads, and other big data applications. These kernels are often run interchangeably and continually across the lifetime of processors, especially in server machines. All numbers and results reported in the next section are averaged over benchmark programs unless otherwise specified.

\subsection{Modeling Aging and Mean Time to Failure for M3D}

This section presents an overview of the major aging and wearout effects. While yield rate is more specific to the process technology and devices and affects the system early on, wearout effects impact the entire system significantly across its lifetime. Examples of aging and wearout effects are TDDB [33], NBTI [10], and EM [12]. In fact, most wearout mechanisms exhibit an exponential dependence on temperature [18, 49]. The RAMP approach [50] models the mean time to failure (MTTF) of a processor as a function of failure rates of individual structures on-chip associated with high temperature and proposes to calculate the reliability. Vattikonda et al. present a divide-andconquer-based reliability evaluation that employs a Monte-Carlo-based simulation [48]. As the heart of reliability evaluation in this article is based on Monte Carlo simulations, we adopted the latter. Moreover, as the basis of the developing 3D technology is on silicon transistors, the common aging mechanisms such as NBTI exist and 2D models are widely used for their simulations [27, 34, $54,56]$. All in all, R2D3 is able to decelerate and control non-uniform aging caused by temperature variation through better usage of each resource. Considering that all wearout mechanisms are heavily dependent on temperature and usage, our method can easily be tuned for them.

\section{EVALUATION}

This section demonstrates the use of the proposed framework. We evaluate our fault detection coverage and compare R2D3 against NoRecon, Static, and R2D3-Lite. Each system has eight cores with one core in each layer. The architectural implementation and tests against faults are done for both the in-order OpenSPARC T1 and the OoO RISC-V BOOM core [9] to validate and check differences of the two different architectures. 
Table 2. Results of Physical Design

\begin{tabular}{|l|c|c|c|c|c|c|}
\hline Core & $\begin{array}{c}\text { Total } \\
\text { Area } \\
\left(\mathrm{mm}^{2}\right)\end{array}$ & $\begin{array}{c}\text { Crossbar } \\
\text { Overhead } \\
(\%)\end{array}$ & $\begin{array}{c}\text { Checker } \\
\text { Overhead } \\
(\%)\end{array}$ & $\begin{array}{c}\text { Protected } \\
\text { Area } \\
(\%)\end{array}$ & $\begin{array}{c}\text { Frequency } \\
\text { Overhead } \\
(\%)\end{array}$ & $\begin{array}{c}\text { Power } \\
(\mathrm{mW})\end{array}$ \\
\hline OpenSPARC T1 & 0.387 & 7.1 & 0.31 & 93 & 8.1 & 250 \\
\hline RV64 BOOM & 0.5 & 6.4 & 0.3 & 96 & 6.1 & 384 \\
\hline
\end{tabular}

\subsection{Physical Design}

We evaluate R2D3 on an eight-core OpenSPARC T1 processor and RISC-V BOOM core [9] as shown in Table 1. The eight cores are connected through a crossbar to an on-chip unified L2 cache. For the NoRecon design, we implement a 3D-stacked processor with each core in a new stack, and for the Static, R2D3-Lite, and R2D3 designs, we additionally insert vertical bus interconnects for reconfiguration. We synthesize and perform place and route of a single core in a commercial $45 \mathrm{~nm}$ SOI technology using Synopsys Design Compiler and Cadence Innovus. Table 2 presents the area and power breakdown based on our physical implementation. Each core has an area of $0.387 \mathrm{~mm}^{2}$ for in-order and $0.5 \mathrm{~mm}^{2}$ for OoO design and operates at a frequency of $1 \mathrm{GHz}$. The area occupied by the crossbars including MIVs, switching logic, and checkers is $7.4 \%$ for in-order and $6.7 \%$ for OoO. Moreover, the frequency decreased by $8.2 \%$ for in-order and 6.1 for OoO designs because of the delay overhead added by the crossbar units and checkers. We report a power of $250 \mathrm{~mW}$ and $384 \mathrm{~mW}$ for in-order and OoO cores excluding the power of register files and caches using Synopsys Primetime, which shows a $6.5 \%$ overhead compared to the NoRecon design. The power derived for each stage is used for thermal simulations as described in Section 5.4.1. There are other smaller components that do not contribute significantly to the area and power and are therefore not listed.

\subsection{Performance in Presence of Faults}

In Figures 9(a) and 9(b), we compare the robustness of R2D3 against the unprotected core (NoRecon) for a different number of faults in our two in-order and OoO eight-core eight-layer design that shows how R2D3 provides better performance beyond one fault. The performance of the design would be similar when having zero or one fault as the number of working cores that can be created is identical. Two is the minimum number of faults when our architecture shows improvement as it can use the leftovers to create working pipelines when they don't happen to the same resource or two stages of the same type in two different layers. After seven faults, R2D3 shows more than $2 \times$ and after 12 faults at least $3 \times$ performance improvement over the NoRecon design. It is worth mentioning that the IPC of R2D3 would decrease to less than $10 \%$ of the original value after 50 faults. R2D3 has a much more graceful degradation in performance with more faults.

R2D3 also does considerably better for the OoO processor (the dotted line in Figure 9(b)) compared the in-order case (the dotted line in Figure 9(a)). The main reason behind it is the difference in number of stages between the two processors. The higher the number of stages means a finer granularity, which leads to a smaller percent of the core being disabled in the case of fault. As R2D3 is taking advantage of the granularity in the pipeline-stage level, it can provide more flexibility and effect for complicated architecture with a higher number of stages. The other reason is that OoO translates to higher IPC and fewer stalls (bubbles) in the pipeline (at the same clock frequency) and, consequently, more usage and worse temperature issues and faster aging. As a result, OoO processors would benefit more when R2D3 is deployed.

\subsection{Fault Detection and Diagnosis}

Based on the fault model discussed in the previous section, we assume that a fault's effect can be observed and verified between two identical pipeline stages. We performed a detailed fault analysis 


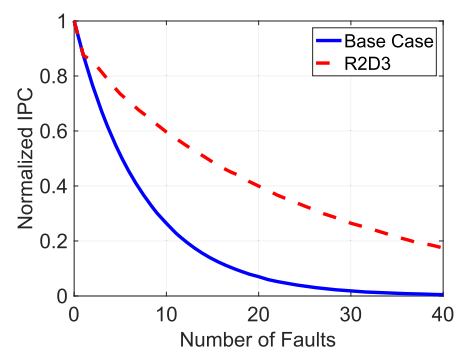

(a)

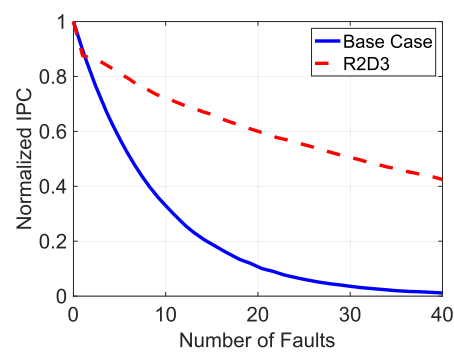

(b)

Fig. 9. Performance of R2D3 with a varying number of concurrent faults for an eight-core eight-layer design with (a) the in-order cores and (b) the OoO cores.

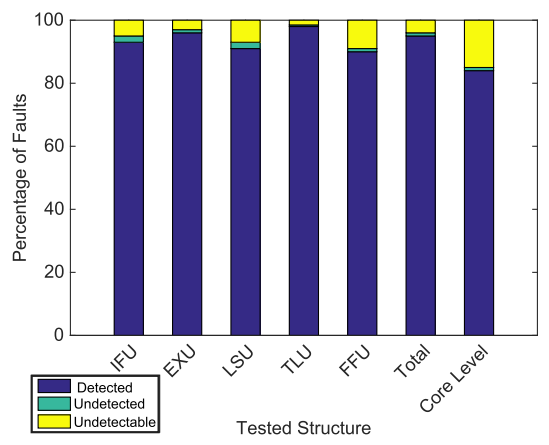

(a)

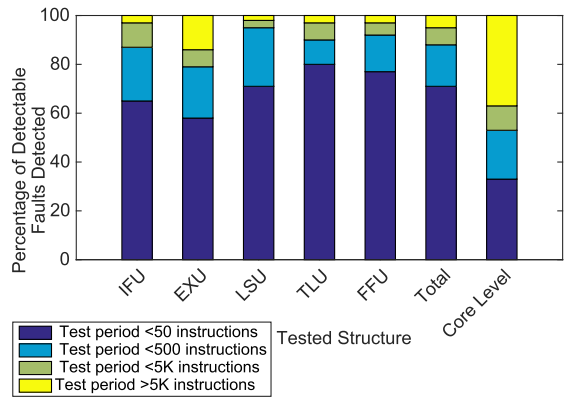

(b)

Fig. 10. (a) Breakdown of different types of faults for each unit. Total illustrates the additive results for all pipeline stages in the stage-level approach, and core-level shows the coverage for solutions that have fault detection at a core granularity. (b) Breakdown of average percentage of detected faults of detectable permanent faults by length of testing period $T_{\text {test }}$.

based on the number of test pattern instructions to determine the protected area covered by the fault detection mechanism as reported in Table 2.

Figure 10(a) breaks down the types of faults for each unit. The column labeled Total illustrates the additive results for all pipeline stages in the stage-level approach, while the Core-Level bar shows the coverage for solutions that target core granularity fault detection. For each case, the bar shows the percentage of detectable faults that are detected, percentage of detectable faults that go undetected when only 10 million ATPG test instructions are run, and percentage of faults that are undetectable regardless of how many ATPG instructions are run. Figure 10(a) shows that the detectable faults (detected plus undetected), which are the target of the detection mechanisms, is $96 \%$ for our pipeline-stage-level architecture (Total) compared to $84 \%$ for a core-level mechanism. As our solution gives better observability over signals at the stage level, it is easier to propagate the faults to observable signals.

5.3.1 Length of Testing Period $\left(T_{\text {test }}\right)$. Figure 10(b) gives the breakdown of the percentage of average detectable permanent faults that are detected within a certain number of instructions by running Monte Carlo fault injection for different applications. For each structure, the bars are divided into several latency stacks from $<50$ to $>5 \mathrm{~K}$ instructions. Figure 10 (b) shows that, on average, $96 \%$ of detectable permanent faults are detected within $5 \mathrm{~K}$ clock cycles (the bar labeled as 

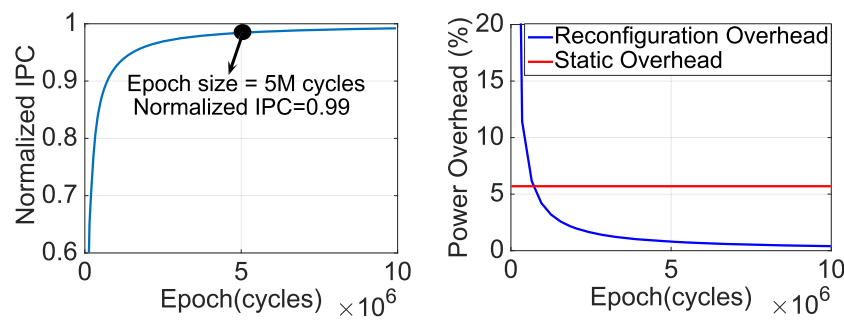

Fig. 11. Worst-case performance degradation (left) and power overhead (right) of reconfiguration policy for varying epoch sizes. For the worst-case scenario, we assume all eight cores are always busy and we have to halt one of them to test and run instructions in parallel. For our epoch size (5M cycles), the performance degradation is only $1 \%$.

Total). This is considerably higher than the $63 \%$ for a core-level architecture. Of course, core-level detection is able to achieve higher than $63 \%$ coverage rates, but possibly with a much longer testing period compared to R2D3 pipeline-stage-level detection. Hence, as $96 \%$ is comparatively high among conventional mechanisms (see Table 3 ), we choose $5 \mathrm{~K}$ cycles as $T_{\text {test }}$, the length of our fault detection testing period at the end of each epoch. By increasing $T_{\text {test }}$, the coverage rate will increase gradually, but this will also result in more power overhead as we are running an idle leftover stage in parallel for a longer time. Thus, there is a trade-off between fault coverage and power overhead. Since the proposed fault detection policy is concurrent, $T_{\text {test }}$ does not cause any performance overhead.

5.3.2 Power Overhead and Epoch Size. Figure 11 (right) shows the power overhead of the proposed mechanism for different epoch sizes $\left(T_{\text {epoch }}\right)$. The total number of stacked cores is kept at eight, the size of the $T_{\text {test }}$ is $5 \mathrm{~K}$ cycles, and 100,000 random fault cases are tested exhaustively for each point on the $\mathrm{x}$-axis. The power penalty is less than $2 \%$ when the epoch size is larger than $2 \mathrm{M}$ cycles. For an epoch size of $5 \mathrm{M}$ cycles, the dynamic power overhead is less than $1 \%$, and thus we select this as our final epoch size. In our design each resource will be checked for permanent faults every $5 \mathrm{M}$ cycles or $5 \mathrm{~ms}$ as our targeted frequency is $1 \mathrm{GHz}$. This determines how $T_{\text {sched }}$ is set, which is the scheduling time for the aging-aware mechanism as well. As NBTI degradation is independent of frequency stress and recovery phases [23,57], we chose our reconfiguration frequency to optimize power and performance overhead and also fault coverage.

5.3.3 Worst-Case Overhead. In our proposed fault detection scheme, no performance penalty is incurred, since only idle leftover resources are utilized. But to test the performance overhead of our solution, we design a worst-case scenario when all cores are working in parallel continuously. Figure 11 (left) shows the normalized IPC of a variety of epoch sizes when all cores are always running. In this case, we need to temporarily suspend one of the cores at the end of each epoch for fault detection of the other ones, which would reduce performance. When the epoch size is $5 \mathrm{M}$ cycles, this IPC overhead is $0.8 \%$. The red graph shows the static power overhead, which is almost constant at $5.7 \%$. Overall, our mechanism has a $6.5 \%$ power overhead when the epoch size is $5 \mathrm{M}$ cycles. As discussed in Section 3.1, the MUX-based crossbar has a fixed channel width, so the delay of transferring an instruction from one stage to the next can take place with $8.2 \%$ frequency overhead within the same clock cycle of pipeline stage execution.

\subsection{Lifetime Management}

This section evaluates the thermal effects, $V_{t h}$ degradation, and the lifetime and performance of R2D3. 


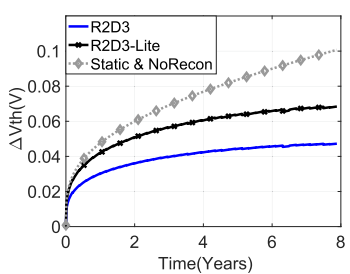

(a)

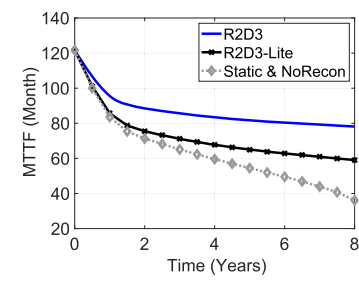

(b)

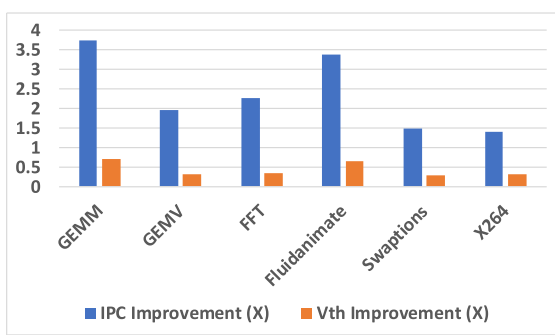

(c)

Fig. 12. Lifetime management evaluation over a period of 8 years: (a) $V_{t h}$ degradation for NoRecon, Static, R2D3-Lite, and R2D3; (b) Mean Time To Failure for NoRecon 3D, Static, R2D3-Lite, and R2D3; (c) IPC and $V_{t h}$ improvement for a subset of benchmark suits.
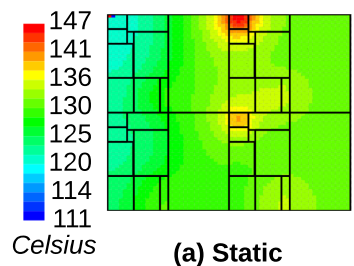

(a) Static

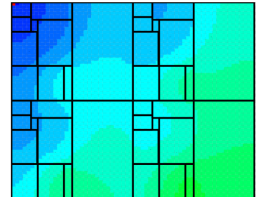

(b) R2D3-Lite

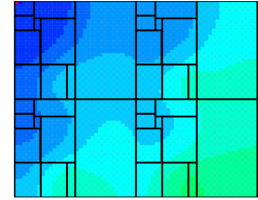

(c) R2D3

Fig. 13. Average temperature map of the hottest layer (farthest from the heat-sink) when running different workloads in a loop for (a) Static 3D, (b) R2D3-Lite, and (c) R2D3.

5.4.1 Thermal Simulation. We computed the thermal impact of the MIVs by assuming a homogeneous via distribution on the die and the combined resistivity of the interface material based on the MIV density. Although R2D3 is not designed to directly deal with hotspots, it is effective in regulating the temperature by managing the usage of hardware resources based on their temperature profile. It prioritizes utilization of resources that are closer to heat-sink and are less susceptible to higher temperature (and, consequently, faster aging). Figure 13 shows the average temperature map of the hottest layer (farthest from the heat-sink) while running different workloads in a loop for Static 3D, R2D3-Lite, and R2D3. R2D3 and R2D3-Lite show up to $33^{\circ} \mathrm{C}$ and $24^{\circ} \mathrm{C}$ reductions in average temperature over Static.

5.4.2 NBTI-based $V_{t h}$ Degradation. We evaluate the $V_{t h}$ degradation caused by the NBTI effect across NoRecon, Static, R2D3-Lite, and R2D3 policies over a period of 8 years as shown in Figure 12(a). Although Static has the capability to adaptively repair the system by routing around failed stages to salvage performance, NoRecon and Static degrade identically as neither of them have protections against $V_{t h}$ degradation and will age at the same rate. In contrast, R2D3-Lite and R2D3 are able to reduce the NBTI effect by $31 \%$ and $53 \%$ over NoRecon and Static. This is because, unlike Static, which only uses a chosen set of pipeline stages that are only reconfigured upon a fault, R2D3-Lite and R2D3 manage to balance usage of units across the 3D fabric by swapping stressed stages with leftovers. Furthermore, since R2D3 differentiates between stages on different layers, implicitly distinguishing the location of cores, we attain an additional $30 \%$ lower $V_{t h}$ degradation over R2D3-Lite.

5.4.3 Lifetime and Performance Assessment. Figure 12(b) shows the MTTF comparison between the multicore systems NoRecon, Static, R2D3-Lite, and R2D3 over a period of 8 years. The results indicate that assigning relatively lighter workloads on NBTI-stressed devices is beneficial for the 


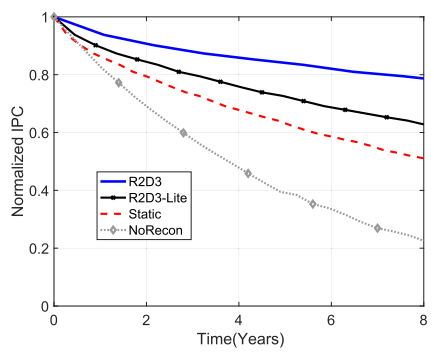

(a)

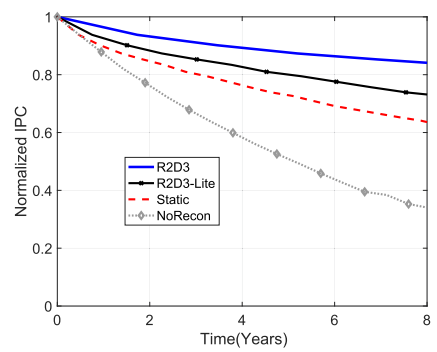

(b)

Fig. 14. Instructions per cycle (IPC) of NoRecon 3D, Static, R2D3-Lite, and R2D3 averaged over different workloads. In all cases, R2D3 shows much more graceful degradation. (a) In-order cores. (b) OoO cores.

recovery of NBTI stress, leading to extended device lifespan. On average, R2D3 and R2D3-Lite demonstrate 2.16× and 1.63× improvements in MTTF degradation over NoRecon and Static in 8 years. This improvement in lifetime also leads to fault reduction, increased number of working resources, and consequently higher performance over an extended period of time. The lifetime numbers in this article are worse compared to a conventional 2D circuit at $45 \mathrm{~nm}$ because we are modeling an eight-layer 3D circuit with much worse thermal hotspots.

We used our evaluation framework described in Section 4 and employed a Monte Carlo simulation using an area-equivalent fault model to compare the performance of our design. Figure 14 illustrates the performance in terms of instructions per cycle (IPC) over a period of 8 years averaged over all workloads. Static, R2D3-Lite, and R2D3 show improvements over NoRecon, as they are able to reconfigure in the case of fault occurrences. On average, for the three workloads, R2D3 improves the performance by $78 \%$ in comparison to NoRecon over the 8 -year period. Moreover, R2D3 and R2D3-Lite improve performance by $21 \%$ and $11 \%$ over Static. Better reconfiguration and usage of resources based on the locality of 3D fabric leads to decelerated aging rates and mitigated NBTI effects, ultimately improving $V_{t h}$ degradation and performance. illustrates the performance in terms of IPC and $V_{t h}$ for a subset of the workloads that have the highest and lowest improvements after a period of 8 years. GEMV and Fluidanimate achieve higher performance improvement in comparison to the other two workloads, especially Swaptions and X264. What distinguishes GEMV and Fluidanimate from the rest is their highly parallel nature of execution. They exhibit higher utilization, power, and temperature and consequently incur higher aging and failure rates. They also benefit more from the extra cores that R2D3 is able to revive using its reconfigurable fabric. This shows that the R2D3 exhibits higher effectiveness for more highly parallel architectures and workloads.

5.4.4 Cluster Size in R2D3. In this section we analyze the impact of increasing the number of layers on area and propagation delay of the interconnect switches. To ascertain the maximum number of layers that can be efficiently stacked together to form a cluster, we evaluated reliability and overhead over varying cluster sizes. Increasing cluster size improves reliability by providing a larger pool of leftovers that can connect and create a working pipeline, but it negatively affects the area footprint and interconnect's propagation delay and system's frequency.

Figure 15 shows the IPC for different cluster sizes when the total stacked cores are kept at 16 . The experiment shows the benefit in reliability by increasing the cluster size, although the gain starts to saturate with the increasing number of cores in a cluster beyond eight layers. This is so because there are enough resources in each cluster to create virtual pipelines and the effect of adding more resources to this pool gets more marginal. 


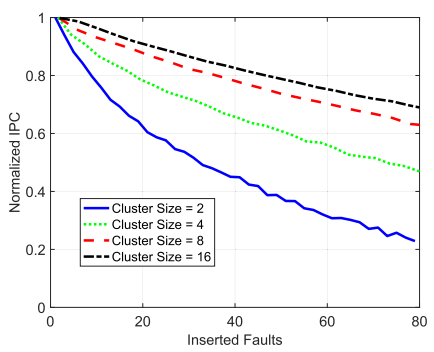

(a)

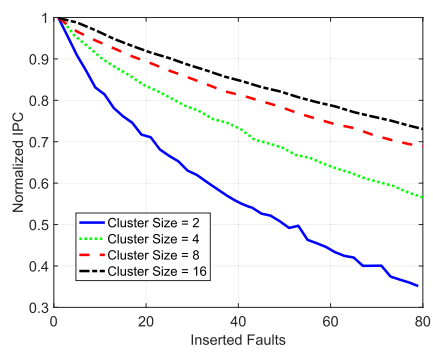

(b)

Fig. 15. Average IPC when cluster size is increasing. The total stacked cores are kept at 16 and 10,000 random fault cases are tested for each point of the x-axis. (a) In-order cores. (b) OoO cores.

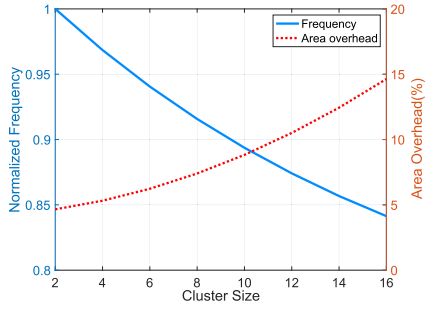

(a)

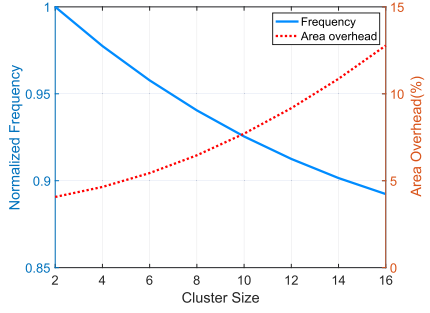

(b)

Fig. 16. Frequency and area of R2D3 for a varying number of 3D layers in a cluster, using the proposed interconnect solution. As the number of cluster size increases, the area overhead increases due to larger crossbars and more MIVs and the frequency will decline due to an increased number of layers to cross within a clock cycle, larger crossbars, and more MIVs. (a) In-order cores. (b) OoO cores.

The second important factor in determining the cluster size is the wire the delay and area overhead caused by crossbars and vertical connections in cluster. Based on the results from Figures 15 and 16, we set the cluster size to 8 as the gain in reliability starts to fade away and the overhead in frequency and area starts to diminish the achieved gains. Figure 16 shows the area and frequency vs. the total cores stacked or the number of slices grouped at a range of cluster sizes. As the number of cores in a cluster increases, the area overhead will increase because of the larger crossbars and more vertical lines required to connect the stages in these cores vertically. The working frequency is decreasing as the number of stacked cores increases because of the delay overhead of the larger crossbars and farther cores in up and bottom of the stacked structure, which have to cross more layers to communicate. The results from Figures 15 and 16 show that considering cluster size as 8 is the best option as the gain in reliability starts to fade away but the overhead in frequency and area starts to dominate the achieved gains.

\section{RELATED WORK}

Table 3 compares the features and performance of R2D3 with related prior work. Generally, we categorize previous solutions into fault detection, diagnosis, repair, and lifetime management (prevention) techniques. Our technique is the only work that addresses all four pillars and achieves comparable or better metrics than previous solutions.

\subsection{Fault Detection and Diagnosis}

Mission critical and high-availability commercial systems ensure high reliability by using modular redundant configurations but invest large portions of Silicon area and incur performance 
Table 3. Feature Comparison Matrix: Fault Detection and Diagnosis, Repair, and Prevention (Lifetime Management) Are the Key Features Required for a Reliable Solution, Which Is Satisfied Only by R2D 3 at a Low Cost

\begin{tabular}{|c|c|c|c|c|c|c|c|c|c|}
\hline \multirow{2}{*}{$\begin{array}{l}\text { Reliabilty } \\
\text { Solutions }\end{array}$} & \multirow{2}{*}{ Granularity } & \multicolumn{2}{|c|}{ Fault Diagnosis } & \multirow{2}{*}{ Fault Repair } & \multicolumn{2}{|c|}{ Lifetime Management } & \multirow{2}{*}{$\begin{array}{l}\text { Performance } \\
\text { Cost }(\%)\end{array}$} & \multirow{2}{*}{$\begin{array}{c}\text { Area Cost } \\
(\%)\end{array}$} & \multirow{2}{*}{$\begin{array}{r}\text { Power } \\
\text { Cost }(\%) \\
\end{array}$} \\
\hline & & Detection & Coverage (\%) & & Technique & Enhancement (\%) & & & \\
\hline ARGUS [36] & Core & $\checkmark$ & 98 & & & & 3.9 & 17.0 & N.R. \\
\hline OnlineTesting [46] & Pipeline stage & $\checkmark$ & 89 & & & & 18.0 & 5.9 & N.R. \\
\hline ACE [11] & Core & $\checkmark$ & 99 & & & & 5.5 & 5.8 & 4.0 \\
\hline mSWAT [22] & Core & $\checkmark$ & 95 & & & & N.R. & N.R. & N.R. \\
\hline DIVA [5] & Core & $\checkmark$ & N.R. & & & & 2.6 & N.R. & N.R. \\
\hline CoreCannibal [45] & Pipeline Stage & & & $\checkmark$ & & & 12.0 & 3.5 & N.R. \\
\hline 3DFAR [7] & Pipeline stage & & & $\checkmark$ & & & 5.0 & 7.0 & N.R. \\
\hline StageNet [20] & Pipeline stage & & & $\checkmark$ & & & 33.0 & 17.0 & 16.0 \\
\hline Viper [42] & Pipeline stage & & & $\checkmark$ & & & 24.0 & 8.0 & N.R. \\
\hline Cobra [41] & Pipeline stage & & & $\checkmark$ & & & 3.0 & N.R. & N.R. \\
\hline NBTI 3D [35] & Core & & & & $\checkmark$ & Performance: 2.4 & 9.0 & N.R. & N.R. \\
\hline Bubblewrap [28] & Core & & & & $\checkmark$ & $\begin{array}{l}\text { Frequency: } 16 \% \\
\text { Throughput: } 30 \%\end{array}$ & N.R. & N.R. & up to 90.0 \\
\hline $\begin{array}{l}\text { NBTI } \\
\text { Multicore [53] }\end{array}$ & Core & & & & $\checkmark$ & $\begin{array}{l}\text { Failure: } 20 \% \\
\text { MTTF: } 30 \%\end{array}$ & 6.0 & N.R. & N.R. \\
\hline Facelift [55] & Core & & & & $\checkmark$ & $\begin{array}{l}\text { Frequency: } 14 \% \\
\text { Lifetime: } 8 \%\end{array}$ & N.R. & N.R. & N.R. \\
\hline Proactive [38] & Core & & & & $\checkmark$ & Lifetime: $63 \%$ & 2.0 & N.R. & N.R. \\
\hline Artemis [44] & Core & & & & $\checkmark$ & Performance: $25 \%$ & 2.0 & N.R. & N.R. \\
\hline R2D3 [this work] & Pipeline stage & $\checkmark$ & 96 & $\checkmark$ & $\checkmark$ & $\begin{array}{l}\text { Performance: } 78 \% \\
\text { Lifetime: } 116 \%\end{array}$ & 8.2 & 7.4 & 6.5 \\
\hline
\end{tabular}

${ }^{*}$ N.R. stands for Not Reported.

penalties. Moreover, due to the coarse granularity of redundancy, such systems would still be unable to perform reliably when every processor is subjected to a high fault rate. Some research has focused on developing low-cost fault-tolerant techniques for classic pipelined processors that rely on online testing [46], runtime fault detection [36], or defect isolation [20, 42]. Authors in [46] utilize a microarchitectural checkpointing mechanism that creates coarse-grained epochs of execution, during which distributed online built-in self-test (BIST) mechanisms validate the integrity of the underlying hardware. Unfortunately, these solutions do not scale to complex designs in a high-fault-rate scenario, and StageNet relies on external mechanisms for fault detection and prevention using lifetime management. Viper [42] proposes a service-oriented execution paradigm. Since it is affected by limitations typical of distributed control architectures, its performance and scalability compare poorly against traditional CMPs and cannot detect faults. Fine-grained architectures can break apart the hardware units of classic hard-wired pipelines, dissolving them into a sea of redundant hardware components. However, these solutions [7, 19, 20, 42, 45] suffer from four key drawbacks. First, they do not address fault detection or diagnosis, which are essential for a holistic treatment solution. They assume that the detection and diagnosis are handled by other mechanisms available in the system. This introduces additional overhead, as fault detection and diagnosis at a fine granularity can be expensive [36, 46]. Second, they do not have a prevention policy that considers usage, heat, or aging. Third, the configuration is static until the occurrence of the next fault, leading to non-uniform aging and thermal variations and thus exaggerating runtime failures in 3D systems. Finally, these architectures need dramatic changes to the processor pipeline and control units that makes them complicated and expensive to implement [7].

Utilizing the third dimension can bring ground-breaking opportunities to enhance the performance of integrated systems. In the context of reliability for 3D layouts, R2D3 provides a unified reliability solution with extremely graceful performance degradation, compared to the limited and specialized approaches mentioned above.

\subsection{Lifetime Management Techniques}

Aging is highly dependent on the utilization and operating temperature. System-level techniques take advantage of the application runtime behavior to improve lifetime reliability. Adaptive voltage scaling (AVS) is an architecture-level technique proposed to mitigate aging in modern 
processors. Facelift [55] is a specific application of dynamic voltage scaling (DVS) in which the supply voltage only adapts once during the lifetime of a processor to switch it from a slow aging mode to a high-speed mode. Bubblewrap [28] uses techniques based on Facelift to enhance performance in a multi-core processor. Artemis [44] is an aging-aware application mapping and DVS scheduling framework that considers the PDN-aging of 3D Network-on-Chip (NoC)-based CMPs.

All of these works only try to mitigate the effects caused by aging early on, instead of reducing the aging process over time. The effect is limited, as when the supply voltage increases to counteract aging, the $V_{t h}$ degradation soon converges to that found in the guardbanded case [44]. In contrast, we show that R2D3 reduces the aging through its smart reconfiguration policy based on the activity and temperature.

\subsection{Comparison to the Frankenstein Solutions}

While some solutions may seem to incur a lower overhead in a particular category, they do not provide all the mechanisms R2D3 does and lose out in other categories. If we forcefully combine multiple solutions together, in a Frankenstein method, then we will see major drawbacks in overhead even if we ignore compatibility issues. For example, if we want to create a combined solution with the highest coverage and best lifetime enhancement, including fault repairing, we would combine [38], [7], and [11]. Not only is this impossible since [11] and [7] are incompatible, but also it would give a $12.5 \%$ performance and $12.8 \%$ area overhead without even considering power. R2D3 incurs much lower overheads in comparison to this Frankenstein solution.

\section{CONCLUSIONS}

In this article, we proposed R2D3, a solution for the reliability of multi-core processors. R2D3 is the first end-to-end dynamic aging-aware framework built on a fine-grained fault-tolerant reconfigurable architecture to detect faults and eliminate/remedy NBTI effects at a marginal performance cost. We proposed a low-cost framework for fault detection by parallel re-execution of instructions on leftovers that achieves a $96 \%$ coverage rate. Considering temperature variation across a 3D chip and its implications on $V_{t h}$ degradation at different cores in different layers, we showed significant reduction of NBTI degradation by swapping in leftover pipeline stages to provide an opportunity for the stressed resources to partially recover $V_{t h}$. Our evaluation, based on performance measurements on a physical design of an OpenSPARC T1 processor over a period of 8 years, showed that R2D3 reduces $V_{t h}$ degradation by $53 \%$ over NoRecon and Static. Furthermore, R2D3 achieved a $21 \%$ increase in IPC along with a $2.16 \times$ improvement in lifetime over Static, while incurring a marginal $7.4 \%$ area and $6.5 \%$ power overhead in comparison to NoRecon. Our analysis for a worst-case scenario, where there are no idle or leftover stages, showed less than a $8.2 \%$ performance overhead.

\section{REFERENCES}

[1] SAMUEL K. MOORE/IEEE Spectrum. First 3D Nanotube and RRAM ICs Come Out of Foundry. https://spectrum.ieee. org/nanoclast/semiconductors/devices/first-3d-nanotube-and-rram-ics-come- out-of-foundry.

[2] Tutu Ajayi, Sumanth Kamineni, Yaswanth K. Cherivirala, Morteza Fayazi, Kyumin Kwon, Mehdi Saligane, Shourya Gupta, Chien-Hen Chen, Dennis Sylvester, David Blaauw, Ronald Dreslinski, Benton Calhoun, and David D. Wentzloff. 2020. An open-source framework for autonomous SoC design with analog block generation. In 2020 IFIP/IEEE 28th International Conference on Very Large Scale Integration (VLSI-SOC'20). 141-146. https://doi.org/10.1109/VLSISOC46417.2020.9344104

[3] Aporva Amarnath, Javad Bagherzadeh, Jielun Tan, and Ronald G. Dreslinski. 2019. 3DTUBE: A design framework for high-variation carbon nanotube-based transistor technology. In 2019 IEEE/ACM International Symposium on Low Power Electronics and Design (ISLPED'19). 1-6. https://doi.org/10.1109/ISLPED.2019.8824874 
[4] Alon Amid, David Biancolin, Abraham Gonzalez, Daniel Grubb, Sagar Karandikar, Harrison Liew, Albert Magyar, Howard Mao, Albert Ou, Nathan Pemberton, et al. 2020. Chipyard: Integrated design, simulation, and implementation framework for custom SoCs. IEEE Micro 40, 4 (2020), 10-21.

[5] Todd Austin. 1999. DIVA: A reliable substrate for deep submicron microarchitecture design. In Proceedings of the 32nd Annual ACM/IEEE International Symposium on Microarchitecture (MICRO'32). IEEE Computer Society, USA, 196-207.

[6] Javad Bagherzadeh, Aporva Amarnath, Jielun Tan, Subhankar Pal, and Ronald G. Dreslinski. 2020. R2D3: A reliability engine for 3D parallel systems. In 2020 57th ACM/IEEE Design Automation Conference (DAC'20). 1-6. https://doi.org/ 10.1109/DAC18072.2020.9218497

[7] J. Bagherzadeh and V. Bertacco. 2017. 3DFAR: A three-dimensional fabric for reliable multi-core processors. In DATE 2017.

[8] Nathan Binkert, Bradford Beckmann, Gabriel Black, Steven K. Reinhardt, Ali Saidi, Arkaprava Basu, Joel Hestness, Derek R. Hower, Tushar Krishna, Somayeh Sardashti, Rathijit Sen, Korey Sewell, Muhammad Shoaib, Nilay Vaish, Mark D. Hill, and David A. Wood. 2011. The Gem5 simulator. SIGARCH Comput. Archit. News 39, 2 (Aug. 2011), 1-7. https://doi.org/10.1145/2024716.2024718

[9] Christopher Celio, David A. Patterson, and Krste Asanovic. 2015. The Berkeley out-of-order machine (boom): An industry-competitive, synthesizable, parameterized RISC-V processor. EECS Department, University of California, Berkeley, Tech. Rep. UCB/EECS-2015-167.

[10] T. B. Chan, J. Sartori, P. Gupta, and R. Kumar. 2011. On the efficacy of NBTI mitigation techniques. In 2011 DATE.

[11] K. Constantinides, O. Mutlu, T. Austin, and V. Bertacco. 2009. A flexible software-based framework for online detection of hardware defects. IEEE Trans. Comput. 58, 8 (Aug. 2009), 1063-1079. https://doi.org/10.1109/TC.2009.52

[12] F. M. d'Heurle. 1971. Electromigration and failure in electronics: An introduction. Proc. IEEE 59, 10 (Oct. 1971$), 1409$.

[13] D. Dutoit, C. Bernard, S. Chéramy, F. Clermidy, Y. Thonnart, P. Vivet, C. Freund, V. Guérin, S. Guilhot, S. Lecomte, G. Qualizza, J. Pruvost, Y. Dodo, N. Hotelier, and J. Michailos. 2013. A 0.9 pJ/bit, 12.8 GByte/s WideIO memory interface in a 3D-IC NoC-based MPSoC. In 2013 Symposium on VLSI Technology. C22-C23.

[14] Morteza Fayazi, Zachary Colter, Ehsan Afshari, and Ronald Dreslinski. 2021. Applications of artificial intelligence on the modeling and optimization for analog and mixed-signal circuits: A review. IEEE Trans. Circuits Syst. I Regular Papers 68, 6 (2021), 2418-2431. https://doi.org/10.1109/TCSI.2021.3065332

[15] P. Gadfort, A. Dasu, A. Akoglu, Y. K. Leow, and M. Fritze. 2014. A power efficient reconfigurable system-in-stack: 3D integration of accelerators, FPGAs, and DRAM. In 2014 27th IEEE International System-on-Chip Conference (SOCC'14). 11-16. https://doi.org/10.1109/SOCC.2014.6948892

[16] B. Ghavami and M. Raji. 2016. Failure characterization of carbon nanotube FETs under process variations: Technology scaling issues. IEEE Transactions on Device and Materials Reliability 16, 2 (June 2016), 164-171. https://doi.org/10.1109/ TDMR.2016.2543659

[17] S. Gupta, A. Ansari, S. Feng, and S. Mahlke. 2009. Adaptive online testing for efficient hard fault detection. In 2009 IEEE International Conference on Computer Design. 343-349. https://doi.org/10.1109/ICCD.2009.5413132

[18] Shantanu Gupta, Shuguang Feng, Amin Ansari, Jason A. Blome, and Scott A. Mahlke. 2008. StageNetSlice: A reconfigurable microarchitecture building block for resilient CMP systems. 1-10. https://doi.org/10.1145/1450095.1450099

[19] S. Gupta, S. Feng, A. Ansari, and S. Mahlke. 2010. Erasing core boundaries for robust and configurable performance. In 2010 43rd Annual IEEE/ACM International Symposium on Microarchitecture. 325-336.

[20] S. Gupta, S. Feng, A. Ansari, Jason Blome, and S. Mahlke. 2008. StageNet: A reconfigurable fabric for constructing dependable CMPs. In Proceedings of the 2008 International Conference on Compilers, Architectures and Synthesis for Embedded Systems. Association for Computing Machinery, 1-10.

[21] O. Hammami, A. M'zah, and K. Hamwi. 2012. Design of 3D-IC for butterfly NOC based 64 PE-multicore: Analysis and design space exploration. In 2011 IEEE International 3D Systems Integration Conference (3DIC'12). 1-4. https://doi.org/ 10.1109/3DIC.2012.6263029

[22] S. K. S. Hari, M. L. Li, P. Ramachandran, B. Choi, and S. V. Adve. 2009. mSWAT: Low-cost hardware fault detection and diagnosis for multicore systems. In 2009 42nd Annual IEEE/ACM International Symposium on Microarchitecture (MICRO'09). 122-132.

[23] Hyejeong Hong, Jaeil Lim, Hyunyul Lim, and Sungho Kang. 2015. Lifetime reliability enhancement of microprocessors: Mitigating the impact of negative bias temperature instability. ACM Comput. Surv. 48 (Sept. 2015), 1-25.

[24] Ahmad Islam. 2013. Variability and reliability of single-walled carbon nanotube field effect transistors. Electronics 2 (Dec. 2013), 332-367. https://doi.org/10.3390/electronics2040332

[25] ITRS. [n.d.]. 2015 International Technology Roadmap for Semiconductors. http://www.itrs2.net/itrs-reports.html.

[26] L. Jiang, F. Ye, Q. Xu, K. Chakrabarty, and B. Eklow. 2013. On effective and efficient in-field TSV repair for stacked 3D ICs. In Proc. DAC.

[27] Tengfei Jiang, Jay Im, Rui Huang, and Paul S. Ho. 2015. Through-silicon via stress characteristics and reliability impact on 3D integrated circuits. MRS Bull. 40, 3 (2015), 248-256. 
[28] U. R. Karpuzcu, B. Greskamp, and J. Torrellas. 2009. The BubbleWrap many-core: Popping cores for sequential acceleration. In 2009 42nd MICRO.

[29] M. I. Khan, A. R. Buzdar, and F. Lin. 2014. Self-heating and reliability issues in FinFET and 3D ICs. In 2014 12th IEEE International Conference on Solid-State and Integrated Circuit Technology (ICSICT'14). 1-3. https://doi.org/10.1109/ ICSICT.2014.7021443

[30] Dae Kim, Suyoun Kim, and Sung Lim. 2011. Impact of nano-scale through-silicon vias on the quality of today and future 3D IC designs. In Proc. SLIP.

[31] Heewoo Kim, Aporva Amarnath, Javad Bagherzadeh, Nishil Talati, and Ronald G. Dreslinski. 2021. A survey describing beyond Si transistors and exploring their implications for future processors. 7. Emerg. Technol. Comput. Syst. 17, 3 (June 2021), Article 27, 44 pages. https://doi.org/10.1145/3453143

[32] H. T. Kung, Bradley McDanel, and Sai Qian Zhang. 2018. Mapping systolic arrays onto 3D circuit structures: Accelerating convolutional neural network inference. In 2018 IEEE International Workshop on Signal Processing Systems (SiPS'18). IEEE, 330-336.

[33] X. Li, J. Qin, and J. B. Bernstein. 2008. Compact modeling of MOSFET wearout mechanisms for circuit-reliability simulation. IEEE Trans. Device Materials Reliabil. 8, 1 (March 2008), 98-121. https://doi.org/10.1109/TDMR.2008.915629

[34] Y. Li, M. Stucchi, S. Van Huylenbroeck, G. Van Der Plas, G. Beyer, E. Beyne, and K. Croes. 2018. TSV process-induced MOS reliability degradation. In 2018 IEEE International Reliability Physics Symposium (IRPS'18). 5B.5-1-5B.5-5. https: //doi.org/10.1109/IRPS.2018.8353610

[35] C. H. Lin, Y. C. Lu, C. K. Tang, and K. Y. Tsai. 2012. The effect of NBTI on 3D integrated circuits. In 2012 IEEE Electrical Design of Advanced Packaging and Systems Symposium (EDAPS'12). 201-204. https://doi.org/10.1109/EDAPS.2012. 6469435

[36] A. Meixner, M. E. Bauer, and D. Sorin. 2007. Argus: Low-cost, comprehensive error detection in simple cores. In Proc. MICRO.

[37] S. Nomura, M. D. Sinclair, C. Ho, V. Govindaraju, M. de Kruijf, and K. Sankaralingam. 2011. Sampling + DMR: Practical and low-overhead permanent fault detection. In 2011 38th Annual International Symposium on Computer Architecture (ISCA'11). 201-212. https://doi.org/10.1145/2000064.2000089

[38] F. Oboril and M. B. Tahoori. 2012. Reducing wearout in embedded processors using proactive fine-grain dynamic runtime adaptation. In 2012 17th IEEE European Test Symposium (ETS'12). 1-6. https://doi.org/10.1109/ETS.2012.6233012

[39] S. Pal, J. Beaumont, D. Park, A. Amarnath, S. Feng, C. Chakrabarti, H. Kim, D. Blaauw, T. Mudge, and R. Dreslinski. 2018 OuterSPACE: An outer product based sparse matrix multiplication accelerator. In 2018 IEEE International Symposium on High Performance Computer Architecture (HPCA'18). 724-736. https://doi.org/10.1109/HPCA.2018.00067

[40] S. Panth, S. Samal, Y. Yu, and S. Lim. 2014. Design challenges and solutions for ultra-high-density monolithic 3D ICs. In Proc. S3S.

[41] A. Pellegrini and V. Bertacco. 2013. Cobra: A comprehensive bundle-based reliable architecture. In 2013 International Conference on Embedded Computer Systems: Architectures, Modeling, and Simulation (SAMOS’13). 247-254.

[42] Andrea Pellegrini, Joseph Greathouse, and Valeria Bertacco. 2012. Viper: Virtual pipelines for enhanced reliability. In Proc. ISCA

[43] C. S. Premachandran, S. Choi, S. Cimino, T. Tran-Quinn, L. Burrell, and P. Justison. 2018. Reliability challenges for 2.5D/3D integration: An overview. In 2018 IEEE International Reliability Physics Symposium (IRPS'18). 5B.4-1-5B.4-5 https://doi.org/10.1109/IRPS.2018.8353609

[44] V. Y. Raparti, N. Kapadia, and S. Pasricha. 2017. ARTEMIS: An aging-aware runtime application mapping framework for 3D NoC-based chip multiprocessors. IEEE Trans. Multi-Scale Comput. Syst. 3, 2 (April 2017), 72-85.

[45] B. F. Romanescu and D. J. Sorin. 2008. Core cannibalization architecture: Improving lifetime chip performance for multicore processors in the presence of hard faults. In 2008 International Conference on Parallel Architectures and Compilation Techniques (PACT'08). 43-51.

[46] Smitha Shyam, Kypros Constantinides, Sujay Phadke, Valeria Bertacco, and Todd Austin. 2006. Ultra low-cost defect protection for microprocessor pipelines. In Proceedings of the 12th International Conference on Architectural Support for Programming Languages and Operating Systems (ASPLOS XII). Association for Computing Machinery, New York, NY, 73-82. https://doi.org/10.1145/1168857.1168868

[47] Jared C. Smolens, Brian T. Gold, James C. Hoe, Babak Falsafi, and Ken Mai. 2007. Detecting emerging wearout faults. In Proc. of Workshop on SELSE.

[48] JEDEC Specification. 2010. Failure mechanisms and models for semiconductor devices. Paper No. JEP 122 (2010), 2010

[49] J. Srinivasan, S. V. Adve, P. Bose, and J. A. Rivers. 2004. The impact of technology scaling on lifetime reliability. In International Conference on Dependable Systems and Networks, 2004. 177-186. https://doi.org/10.1109/DSN.2004.1311888

[50] Jayanth Srinivasan, Sarita V. Adve, Pradip Bose, and Jude A. Rivers. 2005. Exploiting structural duplication for lifetime reliability enhancement. In ACM SIGARCH Computer Architecture News, Vol. 33. IEEE Computer Society, 520-531.

[51] Miloš Stanisavljević, Alexandre Schmid, and Yusuf Leblebici. 2011. Reliability, faults, and fault tolerance. In Reliability of Nanoscale Circuits and Systems. Springer, 7-18.

ACM Journal on Emerging Technologies in Computing Systems, Vol. 18, No. 1, Article 23. Pub. date: November 2021. 
[52] M. C. Strus, R. R. Keller, and N. Barbosa. 2011. Electrical reliability and breakdown mechanisms in single-walled carbon nanotubes. In 2011 11th IEEE International Conference on Nanotechnology. 715-719.

[53] Jin Sun, Roman Lysecky, Karthik Shankar, Avinash Kodi, Ahmed Louri, and Janet Roveda. 2014. Workload assignment considering NBTI degradation in multicore systems. f. Emerg. Technol. Comput. Syst. 10, 1 (Jan. 2014), Article 4, 22 pages. https://doi.org/10.1145/2539124

[54] T. Tanaka. 2017. 3D-IC technology and reliability challenges. In 2017 17th International Workshop on function Technology (IWfT'17). 51-53. https://doi.org/10.23919/IWJT.2017.7966513

[55] A. Tiwari and J. Torrellas. 2008. Facelift: Hiding and slowing down aging in multicores. In 2008 41st IEEE/ACM Symposium on Microarchitecture.

[56] A. Tsiara, X. Garros, C. V. Lu, C. Fenouillet-Béranger, P. Batude, R. Gassilloud, F. Martin, O. Faynot, G. Ghibaudo, and G. Reimbold. 2017. Reliability analysis on low temperature gate stack process steps for 3D sequential integration. In 2017 IEEE SOI-3D-Subthreshold Microelectronics Technology Unified Conference (S3S'17). 1-3. https://doi.org/10.1109/ S3S.2017.8309219

[57] R. Vattikonda, Wenping Wang, and Yu Cao. 2006. Modeling and minimization of PMOS NBTI effect for robust nanometer design. In 2006 43rd DAC.

[58] P. Vivet, Y. Thonnart, R. Lemaire, C. Santos, E. Beigné, C. Bernard, F. Darve, D. Lattard, I. Miro-Panadés, D. Dutoit, F. Clermidy, S. Cheramy, A. Sheibanyrad, F. Pétrot, E. Flamand, J. Michailos, A. Arriordaz, L. Wang, and J. Schloeffel. 2017. A $4 \times 4 \times 2$ homogeneous scalable 3D network-on-chip circuit with $326 \mathrm{MFlit} / \mathrm{s} 0.66 \mathrm{pJ} / \mathrm{b}$ robust and fault tolerant asynchronous 3D links. IEEE F. Solid-State Circuits 52, 1 (Jan. 2017), 33-49. https://doi.org/10.1109/JSSC.2016.2611497

[59] S. Wang, M. B. Tahoori, and K. Chakrabarty. 2015. Defect clustering-aware spare-TSV allocation for 3D ICs. In 2015 IEEE/ACM International Conference on Computer-Aided Design (ICCAD'15). 307-314. https://doi.org/10.1109/ICCAD. 2015.7372585

[60] C. Yang, C. Chou, and J. Li. 2013. A TSV repair scheme using enhanced test access architecture for 3D ICs. In Asian Test Symposium. 7-12.

[61] Runjie Zhang, Mircea Stan, and Kevin Skadron. 2015. HotSpot 6.0: Validation, acceleration and extension. University of Virginia, Tech. Rep. CS-2015-04.

[62] Tiansheng Zhang, Jie Meng, and Ayse K. Coskun. 2015. Dynamic cache pooling in 3D multicore processors. f. Emerg. Technol. Comput. Syst. 12, 2 (Sept. 2015), Article 14, 21 pages. https://doi.org/10.1145/2700247

[63] Wangyuan Zhang and Tao Li. 2008. Microarchitecture soft error vulnerability characterization and mitigation under 3D integration technology. In 2008 41st IEEE/ACM International Symposium on Microarchitecture. 435-446. https://doi. org/10.1109/MICRO.2008.4771811

Received October 2020; revised July 2021; accepted August 2021 